\title{
ON SOME EXTREMAL FUNCTIONS AND THEIR APPLICATIONS IN THE THEORY OF ANALYTIC FUNCTIONS OF SEVERAL COMPLEX VARIABLES( $\left.{ }^{1}\right)$

\author{
BY \\ JOZEF SICIAK
}

1. Introduction. Let $E$ be a bounded closed set in the space $C^{n}$ of $n$-complex variables $z=\left(z_{1}, \ldots, z_{n}\right)$. Let $b(z)$ be a real function defined and bounded on $E$. In the following we define an extremal function $\Phi(z, E, b), z \in C^{n}$, depending on $E$ and $b$. For this purpose we introduce a triangular array of extremal points $\left\{\gamma_{k}^{(v)}\right\}$ of $E$. In the case that $b(z)$ is lower semicontinuous, the formal definition of the points $\gamma_{k}^{(v)}$ is analogous to the definition of Fekete-Leja's point of a plane set. In the case that $E$ is in $C^{1}$ and $b(z) \equiv 0$, the points $\gamma_{k}^{(v)}$ are exactly Fekete's points of $E$ (see $\left(5.3^{\prime}\right)$ ).

In the case of one complex variable, the function $\log \Phi(z, E, 0)$ is a generalized Green's function for the unbounded component of $C E$ with pole at $\infty$. It is well known that the Green's function plays the primary role in the theory of interpolation and approximation of holomorphic functions of one variable by polynomials (see [27]). It turns out that the function $\Phi(z, E, 0), z \in C^{n}$, also plays a quite similar role in the theory of interpolation and approximation of holomorphic functions of several variables by polynomials. For instance, one can obtain the Bernstein-Walsh inequality

$$
\left|P_{v}(z)\right| \leqq M \Phi^{v}(z, E, 0), \quad z \in C^{n}, \quad M=\max _{z \in E}\left|P_{v}(z)\right|
$$

$P_{v}(z)$ being an arbitrary polynomial of order $v, v=0,1, \ldots$ This inequality is useful in the proof of the following theorem: If $\Phi(z, E, 0)$ is continuous in $C^{n}$ and $E_{R}$ is given by

$$
E_{R}=\{z \mid \Phi(z, E, 0)<R\}, \quad R>1
$$

then the necessary and sufficient condition that function $f(z)$ be holomorphic in $E_{R}$ and not continuable to holomorphic (single-valued) function in any $E_{R^{\prime}}$, $R^{\prime}>R$, is that

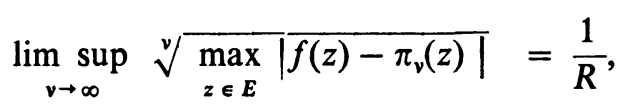

where $\pi_{v}(z)$ denotes a polynomial of order $v$ of the Tchebycheff best approximation to $f(z)$ on $E$.

Received by the editors September 29, 1961.

(1) This work was supported in part by National Science Foundation Grant Number 10375 at Stanford University. 
We also show that polynomials $\mathscr{L}_{v}(z, f)$ found by interpolation of $f(z)$ at the extremal points of $E$ (with respect to $b(z) \equiv 0$ ) converge maximally to $f(z)$.

We prove that if $E$ is a Cartesian product of plane sets $E_{1}, E_{2}, \ldots, E_{n}$, any one of which has positive logarithmic capacity, then

$$
\Phi(z, E, 0) \equiv \max \left\{\Phi\left(z_{1}, E_{1}, 0\right), \ldots, \Phi\left(z_{n}, E_{n}, 0\right)\right\}, \quad z \in C^{n} .
$$

This equality implies that approximation or interpolation by polynomials to the function $f(z)=f\left(z_{1}, \ldots, z_{n}\right)$ holomorphic in the Cartesian product of plane sets reduces, in principle, to approximation or interpolation in each variable separately.

For instance, if $E=E_{1} \times \ldots \times E_{n}$, then (*) is a necessary and sufficient condition that the function $f(z)$ be holomorphic in the Cartesian product $E_{1 R} \times E_{2 R} \times$ $\ldots \times E_{n R}$, where $E_{k R}=\left\{z_{k} \mid \Phi\left(z_{k}, E_{k}, 0\right)<R\right\}$. In the case that $E_{k}, k=1,2, \ldots, n$, is a line segment, this fact has been proved by a different method in [17].

In the last section we prove that if $\Phi(z, E, 0)$ is finite at any point of $C^{n}$, then there exists a limit

$$
u(z, E, b)=\lim _{\lambda \downarrow 0} \frac{1}{\lambda} \log \frac{\Phi(z, E, \lambda b)}{\Phi(z, E, 0)}, \quad z \in C^{n} .
$$

If $E$ is a plane set of positive capacity, then the function $u(z, E, b)$ is harmonic in $C E$. In the case that $E$ is a Jordan curve and $b(z)$ is continuous, the function $u(z, E, b)$ has been proved in [8] and [13] to be a solution of the Dirichlet problem for the interior of $E$ with boundary values $b(z)$. This result has been generalized in [21] as follows. If $E$ is the boundary of a domain $D$ which contains the point $\infty$ in its interior, the function $u(z, E, b)$ is a generalized solution of the Dirichlet problem for any component of $C E$ with boundary values $b(z)$ (continuous or not)

In the last section of this paper the connection of $u(z, E, b)$ with Bremermann's solution (see [4]) of the Dirichlet problem for plurisubharmonic functions in $C^{n}$ and with domains of uniform convergency of Hartogs' series has been established. In particular, we have proved the following. If $E$ is a Silov boundary with respect to polynomials of a bounded domain $D$ such that there exists a decreasing sequence of domains of holomorphy $\left\{D_{v}\right\}$ convergent to $\bar{D}$,

$$
D_{v} \supset D_{v+1} \supset \bar{D}, \quad v=1,2, \ldots, \quad D_{v} \rightarrow \bar{D},
$$

and if any function holomorphic in $\bar{D}$ can be approximated by polynomials uniformly in $\bar{D}$, then the function

$$
u^{*}(z, E, b)=\limsup _{z^{\prime} \rightarrow z} u\left(z^{\prime}, E, b\right), \quad z \in D,
$$

is an upper envelope of all functions $V(z)$ plurisubharmonic in $\bar{D}$ which are less or equal to $b(z)$ on $E$.

Roughly speaking, this means that for bounded polynomially convex domains the function $u^{*}(z, E, b)$ is equal to Bremermann's function. 
The functions $\log \Phi^{*}(z, E, b)$, for $z \in C^{n}$, and $u^{*}(z, E, b)$, for $z \in D$, are plurisubharmonic and therefore are members of some of Bergman's extended classes $[2 ; 3]$. In the case of $C^{1}$, the functions $\log \Phi(z, E, b)$ and $u(z, E, b)$ are harmonic at any finite point outside of $E$ and therefore are continuous there. The natural question of the continuity of $\Phi^{*}(z, E, b)$ or $u^{*}(z, E, b), z \in C^{n}$ (or of the generalized solution of Dirichlet's problem for plurisubharmonic functions) is still open. One knows that in the case of $C^{1}$ there is also a very simple relation between Green's function and Bergman's kernel function. We do not know whether any relation between $\log \Phi^{*}(z, E, 0)$ and the kernel function of several complex variables can be established.

The author wishes to express his gratitude to Professor F. Leja for his inspiration and to Mr. A. Hurd for his help in preparing the manuscript.

2. Lagrange interpolation formulae for polynomials of $n$ complex variables $\left({ }^{2}\right)$. Let $P_{v}(z)=P_{v}\left(z_{1}, \ldots, z_{n}\right)$ be a polynomial of degree $v$

$$
P_{v}(z)=\sum_{k_{1}+\ldots+k_{n} \leqq v} a_{k_{1} \ldots k_{n}} z_{1}^{k_{1}} \ldots z_{n}^{k_{n}} \text {. }
$$

We shall always assume that a polynomial of degree $v$ is also of degree $v^{\prime}, v^{\prime} \geqq v$. Let

$$
k_{1 l}, \ldots, k_{n l}, \quad l=1,2, \ldots, C_{v+n, n}
$$

denote the sequence of all solutions in nonnegative integers of the inequality $k_{1}+\ldots+k_{n} \leqq v$. Then $P_{v}(z)$ may be written in the form

$$
P_{v}(z)=\sum_{l=1}^{v_{*}} a_{k_{1}} \ldots k_{n l} z_{1}^{k_{11}} \ldots z_{n}^{k_{n l}}, \quad v_{*}=^{d f} C_{v+n, n}
$$

Let $p^{(v)}=\left\{p_{1}, p_{2}, \ldots, p_{v_{*}}\right\}$ be a system of $v_{*}$ points

$$
p_{i}=\left(z_{1 i}, \ldots, z_{n i}\right), \quad i=1,2, \ldots, v_{*}
$$

such that the determinant

$$
V\left(p^{(v)}\right)=\operatorname{det}\left[z_{1 i}^{k_{1} l} z_{2 i}^{k_{2 l} l} \ldots z_{n i}^{k_{n l} l}\right], \quad\left(i, l=1,2, \ldots, v_{*}\right),
$$

is different from zero. We observe that $V\left(p^{(v)}\right)$ is a determinant of the system of linear equations

$$
\sum_{l=1}^{v *} a_{k_{11} k_{2 l} \ldots k_{n l}} z_{1 i}^{k_{11}} z_{2}^{k_{2 l}} \ldots z^{k_{n l}}=b_{i}, \quad i=1,2, \ldots, v_{*},
$$

where $a_{k_{1 l} k_{2 l} \ldots k_{n l}}, l=1,2, \ldots, v_{*}$, are unknowns. Therefore, there is exactly one polynomial $P(z)$ of degree $v$ which takes the value $b_{i}$ at the point $p_{i}$ of system $p^{(v)}$.

A system $\mathrm{p}^{(\nu)}=\left\{p_{1}, \ldots, p_{v_{*}}\right\}$ for which $V\left(p^{(\nu)}\right) \neq 0$ will be called unisolvent with respect to polynomials of degree $v$, or simply unisolvent system of order $v$.

Let us replace the $i$ th row of the determinant (4) by the row

$$
\left[z_{1}^{k_{11}} z_{2}^{k_{21}} \ldots z_{n}^{k_{n 1}}, z_{1}^{k_{12}} z_{2}^{k_{22}} \ldots z_{n}^{k_{n 2}}, \ldots, z_{1}^{k_{1 v_{*}}} z_{2}^{k_{2 v *}} \ldots z^{k_{n_{v *}}}\right] \text {. }
$$

(2) Concerning various interpolation formulas in $C^{n}$ see for instance [22; 24]. 
We shall obtain a new determinant (4), say $V_{i}\left(z, p^{(v)}\right)$, which corresponds to the system of points

$$
\left\{p_{1}, p_{2}, \ldots, p_{i-1}, z, p_{i+1}, \ldots, p_{v_{*}}\right\},
$$

$z$ being an arbitrary point of $C^{n}$. Let

We have

$$
L^{(i)}\left(z, p^{(v)}\right)=\frac{V_{i}\left(z, p^{(v)}\right)}{V\left(p^{(\nu)}\right)}, \quad i=1,2, \ldots, v_{*} .
$$

$$
L^{(i)}\left(p_{j}, p^{(v)}\right)=\delta_{i j}, \quad i, j=1,2, \ldots, v_{*} .
$$

The degree of $L^{(i)}\left(z, p^{(\nu)}\right)$ is equal to $v$. We obtain the following:

LEMMA 1. If $p^{(v)}=\left\{p_{1}, \ldots, p_{v_{*}}\right\}$ is unisolvent system of $v_{v}$ points of $C^{n}$ (i.e., $\left.V\left(p^{(v)}\right) \neq 0\right)$ and $P_{v}(z)$ is an arbitrary polynomial of degree $v$, then

$$
P_{v}(z)=\sum_{i=1}^{v *} P_{v}\left(p_{i}\right) L^{(i)}\left(z, p^{(v)}\right), \quad z \in C^{n}
$$

Formula (8) reduces in the case of $n=1$ to the well-known interpolation formula of Lagrange.

Let $\zeta_{k}^{(v)}=\left\{\zeta_{k 0}, \zeta_{k 1}, \ldots, \zeta_{k v}\right\}$ be a system of $v+1$ points of the $\left(z_{k}\right)$-plane, $k=1,2$, $\ldots, n$, respectively. Let $L^{(i)}\left(z_{k}, \zeta_{k}^{(v)}\right)$ denote the fundamental polynomial (6) of the complex variable $z_{k}$ corresponding to the system $\zeta_{k}^{(v)}$. If $P(z)=P\left(z_{1}, \ldots, z_{n}\right)$ is a polynomial of degree $v$ with respect to any of its variables separately, then by iteration of Lagrange's formula for one variable, we obtain

$$
P(z)=\sum_{i_{1}, i_{2}, \ldots, i_{n}=0}^{v} P\left(\zeta_{1 i_{1}}, \zeta_{2 i_{2}}, \ldots, \zeta_{n i_{n}}\right) L^{\left(i_{1}\right)}\left(z_{1}, \zeta_{1}^{(v)}\right) \ldots L^{\left(i_{n}\right)}\left(z_{n}, \zeta_{n}^{(v)}\right) .
$$

Sometimes it is convenient to have a special interpolation formula for homogeneous polynomials. The simplest way of deriving such a formula is to introduce a system of points unisolvent with respect to homogeneous polynomials. Let $Q_{v}(z)=Q_{v}\left(z_{1}, \ldots, z_{n}\right)$ be a homogeneous polynomial of degree $v$,

$$
Q_{v}(z)=\sum_{k_{1}+\ldots+k_{n}=v} a_{k_{1} \ldots} k_{n} z_{1} \ldots z_{n}^{k_{n}},
$$

where $\Sigma_{k_{1}+\ldots+k_{n}=v}$ denotes summation over all solutions in nonnegative integers of the equation $x_{1}+x_{2}+\ldots+x_{n}=v$. Let

$$
\left(k_{1 l}, k_{2 l}, \ldots, k_{n l}\right), \quad l=1,2, \ldots, v_{0}, v_{0}=C_{v+n-1, n-1},
$$

be a complete sequence of these solutions. Then

$$
Q_{v}(z)=\sum_{l=1}^{v_{0}} a_{k_{11} k_{21} \ldots k_{n l}} z_{1}^{k_{11}} \ldots z_{n}^{k_{n l}} .
$$

Let $p^{(v)}=\left\{p_{1}, \ldots, p_{v_{0}}\right\}$ denote a system of $v_{0}$ points of $C^{n}$ where

$$
p_{i}=\left(z_{1 i}, z_{2 i}, \ldots, z_{n i}\right), \quad i=1,2 \ldots, v_{0},
$$

such that the determinant 


$$
W\left(p^{(v)}\right)=\operatorname{det}\left[z_{1 i}^{k_{1 i} l} z_{2 i}^{k_{2 l} l} \ldots z_{n i}^{k_{n} t}\right], \quad i, l=1,2, \ldots, v_{0},
$$

is different from zero. Let $W_{i}\left(z, p^{(\nu)}\right)$ denote the determinant (11) corresponding to the system $\left\{p_{1}, \ldots, p_{i-1}, z, p_{i+1}, \ldots, p_{v_{0}}\right\}, z$ being an arbitrary point of $C^{n}$. The polynomial

$$
T^{(i)}\left(z, p^{(v)}\right)=\frac{W_{i}\left(z, p^{(v)}\right)}{W\left(p^{(v)}\right)}, \quad i=1,2, \ldots, v_{0},
$$

is, of course, homogeneous and of degree $v$. Moreover

$$
T^{(i)}\left(p_{j}, p^{(v)}\right)=\delta_{i j}, \quad i, j=1,2, \ldots, v_{0},
$$

whence we have.

LeMmA 2. If $p^{(v)}=\left\{p_{1}, \ldots, p_{v_{0}}\right\}, v_{0}=C_{v+n-1, n-1}$, is a system of $v_{0}$ points of $C^{n}$ such that $W\left(p^{(v)}\right) \neq 0$ and if $Q_{v}(z)$ is an arbitrary homogeneous polynomial of degree $v$, then

$$
Q_{v}(z)=\sum_{i=1}^{v_{0}} Q_{v}\left(p_{i}\right) T^{(i)}\left(z, p^{(v)}\right) \quad z \in C^{n} .
$$

\section{Interpolation series of Newton. Let}

$$
\zeta_{k}^{(v)}=\left\{\zeta_{k 0}, \zeta_{k 1}, \ldots, \zeta_{k v}\right\}
$$

be a system of $v+1$ distinct points of the $z_{k}$-plane, $k=1,2, \ldots, n$, respectively. Consider the points in $C^{n}$ given by

$$
p_{i_{1}} \cdots i_{n}=\left(\zeta_{1 i_{1}}, \zeta_{2 i_{2}}, \ldots, \zeta_{n i_{n}}\right),
$$

where $i_{1}, i_{2}, \ldots, i_{n}$ are nonnegative integers such that

$$
i_{1}+i_{2}+\ldots+i_{n} \leqq v .
$$

There are $v_{*}=C_{v+n, n}$ such points. We remember that $v_{*}$ is also the number of coefficients of a polynomial $P_{v}(z), z \in C^{n}$, of degree $v$.

LeMMA 1. Given $v_{*}$ arbitrary complex numbers

$$
b_{i_{1} i_{2} \ldots i_{n}}, \quad i_{1}+\ldots+i_{n} \leqq v,
$$

there exists exactly one polynomial $P_{v}(z)$ of degree $v$ such that

$$
P_{v}\left(p_{i_{1} \ldots i_{n}}\right)=b_{i_{1} \ldots i_{n}}, \quad i_{1}+\ldots+i_{n} \leqq v .
$$

Moreover polynomial $P_{v}$ may be written in an unique way in the form

$$
P_{v}(z)=\sum_{i_{1}+\ldots+i_{n} \leqq v} a_{i_{1} \ldots i_{n}} \prod_{k=1}^{n}\left(z_{k}-\zeta_{k_{0}}\right) \ldots\left(z_{k}-\zeta_{k i_{k}-1}\right),
$$

where by definition we put $\left(z_{k}-\zeta_{k,-1}\right)=1$. 
Proof. At first we shall prove that there is a unique polynomial of the form (4) which satisfies (3). For $n=1$ the formula (4) reduces to the well-known interpolation formula of Newton. For $n=2$ see [22]. We shall use induction. Suppose our theorem to be true for $n-1$ variables and observe that

$$
\begin{aligned}
P_{v}(z)= & \sum_{i_{1}=0}^{v}\left[\sum_{i_{2}+\ldots+i_{n} \leq v-i_{1}} a_{i_{1} i_{2} \ldots i_{n}} \prod_{k=2}\left(z_{k}-\zeta_{k 0}\right) \ldots\left(z_{k}-\zeta_{k i_{k}-1}\right)\right] \\
& \times\left(z_{1}-\zeta_{10}\right) \ldots\left(z_{1}-\zeta_{1 i_{1}-1}\right) \\
= & \sum_{i_{1}=0}^{v} P_{v-i_{1}}\left(z_{2}, \ldots, z_{n}\right)\left(z_{1}-\zeta_{10}\right) \ldots\left(z_{1}-\zeta_{1 i_{1}-1}\right) .
\end{aligned}
$$

Equations (3) may now be written in the form

$$
\begin{aligned}
& b_{0 i_{2} i_{3} \ldots i_{n}}=P_{v}\left(\zeta_{2 i_{2}}, \zeta_{3 i_{3}}, \ldots, \zeta_{n i_{n}}\right), \quad i_{2}+i_{3}+\ldots+i_{n} \leqq v \\
& b_{1 i_{2} i_{3} \ldots i_{n}}=P_{v}\left(\zeta_{2 i_{2}}, \zeta_{3 i_{3}}, \ldots, \zeta_{n i_{k}}\right) \\
& +P_{v-1}\left(\zeta_{2 i_{2}}, \zeta_{3 i_{3}}, \ldots, \zeta_{n i_{n}}\right)\left(\zeta_{11}-\zeta_{10}\right), i_{2}+\ldots+i_{n} \leqq v-1 \\
& b_{v 00 \ldots 0}=P_{v}\left(\zeta_{20}, \zeta_{30}, \ldots, \zeta_{n 0}\right)+\ldots \\
& +P_{0}\left(\zeta_{20}, \ldots, \zeta_{n 0}\right)\left(\zeta_{1 v}-\zeta_{10}\right) \ldots\left(\zeta_{1 v}-\zeta_{1 v-1}\right) \text {. }
\end{aligned}
$$

Due to the induction assumption the first $C_{v+n-1, n-1}$ equations enable us to find all the coefficients $a_{0 i_{2} \ldots i_{n}}, i_{2}+\ldots+i_{n} \leqq v$, the next $C_{v+n-2, n-1}$ equations enable us to find all the coefficients $a_{1 i_{2} \ldots i_{n}}, i_{2}+\ldots+i_{n} \leqq v-1$, provided $a_{0 i_{2} \ldots i_{n}}$ have been found, and so on. We shall find all the coefficients $a_{i_{1} \ldots i_{n}}$, $i_{1}+\ldots+i_{n} \leqq v$, successively. However, these coefficients are uniquely determined by (3). By the way, we see that for any values $b_{i_{1} \ldots i_{n}}, i_{1}+\ldots+i_{n} \leqq v$, there is at least one polynomial $P_{v}(z)$ of degree $v$ such that $P_{v}\left(p_{i_{1} \ldots i_{n}}\right)=b_{i_{1} \ldots i_{n}}$. From the theory of linear equations this implies that the determinant (2.4) corresponding to the points (2) is different from zero. Therefore, there is exactly one polynomial $P_{v}(z)$ of degree $v$, which satisfies (3).

One may easily check that the determinant $\Delta$ of equations $\left(4^{\prime}\right)$ has the form$$
\Delta=\delta \cdot\left(\zeta_{11}-\zeta_{10}\right)^{C_{v+n-2, n+1}}
$$$$
\times\left[\left(\zeta_{12}-\zeta_{10}\right)\left(\zeta_{12}-\zeta_{11}\right)\right]^{c_{v+n-3, n-1}}, \ldots\left[\left(\zeta_{1 v}-\zeta_{10}\right) \ldots\left(\zeta_{1 v}-\zeta_{1 v-1}\right)\right]^{c_{n-1, n-1}},
$$

where $\delta$ depends only on the points of $\zeta_{k}^{(v)}$ for $k=2, \ldots, v$. After elementary transformations we obtain from (5)

where

$$
\Delta=\delta \prod_{s=0}^{v-1}\left[V_{s}\left(\zeta_{1}^{(v)}\right)\right]^{c_{s+n-2, n-2}},
$$

$$
V_{s}\left(\zeta_{1}^{(v)}\right)=\prod_{0 \leqq i<j \leqq v-s}\left(\zeta_{1 j}-\zeta_{1 i}\right) .
$$

Since the systems $\zeta_{k}^{(v)}, k=1,2, \ldots, n$, play an equivalent role in $\left(4^{\prime}\right)$, we have

$$
|\Delta|=\left|\prod_{k=1}^{n} \prod_{s=0}^{v-1}\left[V_{s}\left(\zeta_{k}^{(\nu)}\right)\right]^{C_{s+n-2, n-2}}\right| .
$$


By this formula we have $\Delta \neq 0$, whence we may obtain another proof of Lemma 1.

Now we shall find an explicit formula for the coefficients $a_{l_{1} \ldots l_{n}}$. For this purpose let $D_{k}$ be a domain in the $z_{k}$-plane with a smooth boundary $C_{k}$ oriented positively with respect to $D_{k}$. Suppose that the points (1) lie in $D_{k}, k=1,2, \ldots, n$, respectively. Then by the residue theorem

$$
\begin{aligned}
& \frac{1}{(2 \pi i)^{n}} \int_{c_{1}} \ldots \int_{c_{n}} \frac{P_{v}\left(\zeta_{1}, \ldots, \zeta_{n}\right) d \zeta_{1} \ldots d \zeta_{n}}{\prod_{k=1}^{n}\left(\zeta_{k}-\zeta_{k 0}\right) \ldots\left(\zeta_{k}-\zeta_{k l_{k}}\right)} \\
& =\sum_{i_{1}+\ldots+i_{n} \leqq v} a_{i_{1}} \ldots i_{n} \frac{1}{(2 \pi i)^{n}} \int_{c_{1}} \ldots \int_{C_{n}} \prod_{k=1}^{n} \frac{\left(\zeta_{k}-\zeta_{k 0}\right) \ldots\left(\zeta_{k}-\zeta_{k i_{k}-1}\right)}{\left(\zeta_{k}-\zeta_{k 0}\right) \ldots\left(\zeta_{k}-\zeta_{k l_{k}}\right)} \\
& \cdot d \zeta_{1} \ldots d \zeta_{n}=a_{l_{1} l_{2} \ldots l_{n}} .
\end{aligned}
$$

On the other hand, the first integral in (7), also by the residue theorem, is equal to

whence

$$
\sum_{j_{1}=0}^{l_{1}} \sum_{j_{2}=0}^{l_{2}} \ldots \sum_{j n=0}^{l_{n}} \frac{P_{v}\left(\zeta_{1 j_{1}}, \zeta_{2 j_{2}}, \ldots, \zeta_{n j_{n}}\right)}{\left.\prod_{k=0}^{n}\left(\zeta_{k j_{k}}-\zeta_{k 0}\right) \ldots\right|_{\left(j_{k}\right)} \ldots\left(\zeta_{k j_{k}}-\zeta_{k l_{k}}\right)},
$$

$$
a_{l_{1} \ldots l_{n}}=\sum_{j_{1}=0}^{l_{1}} \ldots \sum_{j=0}^{l_{n}} \frac{b_{j_{1}} \ldots j_{n}}{\left.\prod_{k=0}^{n}\left(\zeta_{k j_{k}}-\zeta_{k 0}\right) \ldots\right|_{\left(j_{k}\right)} \ldots\left(\zeta_{k j_{k}}-\zeta_{k l_{k}}\right)}
$$

where $\left.\right|_{\left(j_{k}\right)}$ means that the factor $\left(\zeta_{k j_{k}}-\zeta_{k j_{k}}\right)$ is omitted.

LEMMA 2. If the function $f(z)$ is holomorphic in the closure of $D=D_{1} \times$ $\ldots \times D_{n}$ and if

$$
\zeta_{k 0}, \zeta_{k 1}, \ldots, \zeta_{k v}, \ldots
$$

is a sequence of different points of $D_{k}, k=1,2, \ldots, n$, respectively, then the series

where

$$
f \sim \sum_{l=0}^{\infty} \sum_{i_{1}+\ldots+i_{n}=l} a_{i_{1} \ldots i_{n}} \prod_{k=1}^{n}\left(z_{k}-\zeta_{k 0}\right) \ldots\left(z_{k}-\zeta_{k i_{k}-1}\right)
$$

$$
a_{i_{1} \ldots i}=\frac{1}{(2 \pi i)^{n}} \int_{c_{1}} \ldots \int_{c_{n}} \frac{f\left(\zeta_{1}, \ldots, \zeta_{n}\right) d \zeta_{1} \ldots d \zeta_{n}}{\prod_{k=1}^{n}\left(\zeta_{k}-\zeta_{k 0}\right) \ldots\left(\zeta_{k}-\zeta_{k i_{k}}\right)}
$$

converges to $f(z)$ at any point $\left(\zeta_{11_{1}}, \zeta_{2 l_{2}}, \ldots, \zeta_{n l_{n}}\right), l_{1}, l_{2}, \ldots, l_{n}=0,1,2, \ldots$

Indeed, the integral on the right hand side of $(10)$ is by the residue theorem equal to the expression on the right hand side of (8), in which $b_{j_{1} \ldots j_{n}}$ has been replaced by $f\left(\zeta_{1 j_{1}}, \ldots, \zeta_{n j_{n}}\right)$. This implies that the polynomial

$$
P_{v}(z)=\sum_{l=0}^{v} \sum_{i_{1}+\ldots+i_{n}=l} a_{i_{1} \ldots i_{n}} \prod_{k=1}^{n}\left(z_{k}-\zeta_{k 0}\right) \ldots\left(z_{k}-\zeta_{k i_{k}-1}\right),
$$

being a partial sum of the series (9), takes the value $f\left(\zeta_{1 i_{1}}, \ldots, \zeta_{n l_{n}}\right)$ at the point $\left(\zeta_{1 l_{1}}, \ldots, \zeta_{n l_{n}}\right)$, provided $v \geqq l_{1}+\ldots+l_{n}$. Thus Lemma 2 is true. 
Remark. Let us observe that the series (9) differes from the multiple Newton's series

$$
\sum_{i_{1}, i_{2} \ldots i_{n}=0}^{\infty} a_{i_{1} \ldots i_{n}} \prod_{k=1}^{n}\left(z_{k}-\zeta_{k 0}\right) \ldots\left(z_{k}-\zeta_{k i_{k}-1}\right)
$$

only by a special method of summation. The series (11) is, of course, also convergent to $f$ at the points $\left(\zeta_{1 l_{1}}, \ldots, \zeta_{n l_{n}}\right), l_{1}, \ldots, l_{n}=0,1,2, \ldots$.

4. Unisolvent sets. We shall say that the set $E \subset C^{n}$ is unisolvent of order $v$ if there is at least one unisolvent system $p^{(v)} \subset E$, i.e., a system such that $V\left(p^{(v)}\right) \neq 0$. If $E$ contains unisolvent systems $p^{(v)}$ of any order $v=0,1, \ldots$, we say that $E$ is unisolvent. It is easily shown that a set $E$ unisolvent of order $v$ is also unisolvent of order $k, k=0,1, \ldots, v-1$. To see this it is enough to consider the generalized Laplace's development of $V\left(p^{(\nu)}\right)$. In the case of one complex variable the determinant $V\left(p^{(v)}\right)$ given by (2.4) is simply a determinant of Vandermonde of order $v$ and therefore any system of $v_{*}=v+1$ different points of $C^{1}$ is unisolvent with respect to polynomials in one variable of degree $v$. In the case of $C^{1}$ the homogeneous polynomials $Q_{v}(z)$ of degree $v$ has the form $Q_{v}(z)=a z_{1}^{v}, a=$ const. Here the problem of unisolvency is trivial.

In the space $C^{n}, n \geqq 2$, the unisolvent systems are not so simply characterized. There are systems $p^{(v)}=\left\{p_{1}, \ldots, p_{v *}\right\}$ and $q^{(v)}=\left\{q_{1}, \ldots, q_{v_{0}}\right\}$ of different points $\in C^{n}$ such that $V\left(p^{(v)}\right)=0$ and $W\left(q^{(v)}\right)=0$, respectively.

It follows from Lemma $1, \S 3$, that if $E$ is a set of $(v+1)^{n}$ points

$$
p_{i_{1} \ldots i_{n}}=\left(\zeta_{1 i_{1}}, \ldots, \zeta_{n i_{n}}\right), \quad 0 \leqq i_{1}, i_{2}, \ldots, i_{n} \leqq v,
$$

where $\zeta_{k 0}, \zeta_{k 1}, \ldots, \zeta_{k v}$ are different, then the system of points (1) which satisfies $i_{1}+\ldots+i_{n} \leqq v$ is unisolvent. In fact, there are at least $[(v+1) !]^{n}$ different unisolvent systems of order $v$ in the set $E$.

COROLlaRY 1. If E contains the Cartesian product of the sequences

$$
\zeta_{k 0}, \zeta_{k 1}, \ldots, \quad k=1,2, \ldots, n ; \quad\left(\zeta_{k i} \neq \zeta_{k J} \text { for } i \neq j\right)
$$

then $E$ is unisolvent with respect to polynomials of $n$ complex variables.

We shall now find the absolute value of the determinant (2.4) which corresponds to the system $p^{(v)}$ of points (1). We denote this determinant by

$$
V\left(\left(\zeta_{1 i_{1}}, \ldots, \zeta_{n i_{n}}\right), \quad i_{1}+\ldots+i_{n} \leqq v\right) .
$$

Let $P_{v}(z)$ be a polynomial of degree $v$ such that

$$
P_{v}\left(p_{i_{1} \ldots i_{n}}\right)=b_{i_{1} \ldots i_{n}}, \quad i_{1}+\ldots+i_{n} \leqq v,
$$

the $b_{i_{1} \ldots i_{n}}$ being arbitrary fixed complex numbers. The determinant of the linear equations (3) with unknowns $a_{i_{1} \ldots i_{n}}$ is equal to (2). The polynomial $P_{v}(z)$ may be written in the form 


$$
\begin{aligned}
P_{v}(z) & =\sum_{k_{1}=0}^{v}\left(\sum_{k_{2}+\ldots+k_{n} \leqq v-k_{1}} a_{k_{1}} \ldots k_{n} z_{2} \quad k_{2} \ldots z_{n}^{k_{n}}\right) z_{1}^{k_{1}} \\
& =\sum_{k_{1}=0}^{v} P_{v-k_{1}}\left(z_{2}, \ldots, z_{n}\right) z_{1}^{k_{1}}
\end{aligned}
$$

where $P_{v-k_{1}}\left(z_{2}, \ldots, z_{n}\right)$ is a polynomial of degree $v-k_{1}$ of the $n-1$ variables $z_{2}, \ldots, z_{n}$. To begin with, let us assume that $\zeta_{10}=0$. Then the equations (3) may be written in the form

$$
P_{v}\left(\zeta_{2 i_{2}}, \ldots, \zeta_{n i_{n}}\right)=b_{0 i_{2} \ldots i_{n}}, \quad i_{2}+\ldots+i_{n} \leqq v
$$

$$
\begin{aligned}
\zeta_{1 i} \sum_{k=0}^{v-1} P_{v-k-1}\left(\zeta_{2 i_{2}}, \ldots, \zeta_{n i_{n}}\right) \zeta_{1 i}^{k}=- & P_{v}\left(\zeta_{2 i_{2}}, \ldots, \zeta_{n i_{n}}\right)+b_{i i_{2}} \cdots i_{n}, \\
& i_{2}+\ldots+i_{n} \leqq v-i, \quad i=1,2, \ldots, v
\end{aligned}
$$

whence the following recurrential formula follows for

$$
V\left(p^{(v)}\right)={ }^{d f} V\left(\left(\zeta_{1 i_{1}}, \ldots, \zeta_{n i_{n}}\right), \quad i_{1}+\ldots+i_{n} \leqq v\right)
$$

(4) $V\left(\left(\zeta_{1 i_{1}}, \ldots, \zeta_{n i_{n}}\right), i_{1}+\ldots+i_{n} \leqq v\right)=V\left(\left(\zeta_{2 i_{2}}, \ldots, \zeta_{n i_{n}}\right), i_{2}+\ldots+i_{n} \leqq v\right)$

$$
\begin{gathered}
\times V\left(\left(\zeta_{1 i_{1}+1}, \zeta_{2 i_{2}}, \ldots, \zeta_{n i_{n}}\right), i_{1}+\ldots+i_{n} \leqq v-1\right) \zeta_{11}^{C_{v+n-2, n-1}} \zeta_{12}^{C_{v+n-3, n-1}} \\
\ldots \zeta_{1 v} c_{n-1, n-1} .
\end{gathered}
$$

Since the determinant $V\left(p^{(v)}\right)$ does not depend on unitary transformation of $C^{n}$ onto itself, it follows that if $\zeta_{10} \neq 0$ then

$$
\begin{aligned}
& V\left(p^{(v)}\right)=V\left(\left(\zeta_{2 i_{2}}, \ldots, \zeta_{n i_{n}}\right), \quad i_{2}+\ldots+i_{n} \leqq v\right) \\
& \left.\times V\left(\zeta_{1 i_{1}+1}-\zeta_{10}, \quad \zeta_{2 i_{2}}, \ldots, \zeta_{n i_{n}}\right), i_{1}+\ldots+i_{n} \leqq v-1\right) \\
& \times\left(\zeta_{11}-\zeta_{10}\right)^{C_{\nu+n-2, n-1}}\left(\zeta_{12}-\zeta_{10}\right)^{C_{\nu+n-3, n-1}} \\
& \ldots\left(\zeta_{1 v}-\zeta_{10}\right)^{C_{n-1, n-1}} \text {. }
\end{aligned}
$$

Since $\left|V\left(p^{(v)}\right)\right|$ is symmetric with respect to $\zeta_{k}^{(v)}, k=0,1,2, \ldots, v$, we obtain from (5)

$$
\left|V\left(\left(\zeta_{1 i_{1}}, \ldots, \zeta_{n i_{n}}\right), i_{1}+\ldots+i_{n} \leqq v\right)\right|=\left|\prod_{k=1}^{n} \prod_{s=0}^{v-1}\left[V_{s}\left(\zeta_{k}^{(v)}\right)\right]^{c_{s+n-2, n-2}}\right|
$$

where

$$
V_{s}\left(\zeta_{k}^{(v)}\right)=\prod_{0 \leqq i<j \leqq v-s}\left(\zeta_{k j}-\zeta_{k i}\right), \quad s=0,1, \ldots, v-1 .
$$

By the way, we have proved that the absolute value of the determinant of the equations (3) is equal to the absolute value of the determinant of the equations (3.4').

Let $Q(z)=Q\left(z_{1}, \ldots, z_{n}\right)$ be a homogeneous polynomial of degree $v$. Then the function 


$$
P\left(z_{2}, z_{3}, \ldots, z_{n}\right)=\left(z_{1}^{0}\right)^{v} \cdot Q\left(1, \frac{z_{2}}{z_{1}^{0}}, \ldots, \frac{z_{n}}{z_{1}^{0}}\right), z_{1}^{0}=\text { const } \neq 0
$$

is an ordinary polynomial in $n-1$ variables of degree $v$.

Corollary 2. A set $E \subset C^{n}$, which is a Cartesian product of a point $z_{1}^{0} \neq 0$ and of systems $\zeta_{k}^{(v)}=\left\{z_{k 0}, z_{k_{1}}, \ldots, z_{k v}\right\}, k=2, \ldots, n$, of different points of the $z_{k^{-}}$ plane, is unisolvent of order $v$ with respect to homogeneous polynomials.

5. Extremal points. Let $E$ be a bounded closed set in $C^{n}$. Let $b(z)$ be a real function defined and bounded on $E$. Given an arbitrary system $p^{(v)}=\left\{p_{1}, \ldots, p_{v *}\right\}$ $\subset E$ of points

$$
p_{l}=\left(z_{1 l}, \ldots, z_{n l}\right), \quad l=1,2, \ldots, v_{*}, \quad v_{*}=C_{v+n, n}
$$

we define $V\left(p^{(v)}, b\right)$ by

$$
V\left(p^{(v)}, b\right)=V\left(p^{(v)}\right) \exp \left[-v \sum_{l=1}^{v_{*}} b\left(p_{l}\right)\right], \quad v=0,1,2, \ldots,
$$

where $V\left(p^{(v)}\right)$ is given by (2.4). Let $\left\{\alpha_{v}\right\}$ be a sequence of real numbers such that

$$
\alpha_{v}>1, \quad v=0,1, \ldots, \quad \text { and } \lim _{v \rightarrow \infty}\left(\alpha_{v}\right)^{1 / v}=1 \text {. }
$$

For any $v=0,1, \ldots$ there is a system

$$
\gamma^{(v)}=\left\{\gamma_{1}^{(v)}, \gamma_{2}^{(v)}, \ldots, \gamma_{v_{*}}^{(v)}\right\}
$$

of points of $E$ such that

$$
\left|V\left(\gamma^{(v)}, b\right)\right|>\alpha_{v}^{-1}\left|V\left(p^{(v)}, b\right)\right|, \quad v=1,2, \ldots,
$$

$p^{(v)}$ being an arbitrary system of $v_{*}$ points of $E$.

The system (3) will be called the $v$ th extremal system of $E$ with respect to $b(z)$, $\left\{\alpha_{v}\right\}$ and $V\left(p^{(v)}\right)$. The points of system (3) will be called the extremal points of order $v$.

If $b(z)$ is lower semicontinuous then $V\left(p^{(v)}, b\right)$ is upper semicontinuous with respect to $p^{(v)}$. Therefore, in that case there is a system

$$
q^{(v)}=\left\{q_{1}^{(v)}, q_{2}^{(v)}, \ldots, q_{v_{*}}^{(v)}\right\}
$$

of points $E$ such that

$$
\left|V\left(q^{(i)}, b\right)\right|=\max _{p^{(v)} \subset E}\left|V\left(p^{(v)}, b\right)\right|, \quad v=0,1,2, \ldots
$$

The points ( $\left.3^{\prime}\right)$ will be called ordinary extremal points of $E$ with respect to $b(z)$ (and $V\left(p^{(v)}\right)$ ). In the case that $E \subset C^{1}$ and $b(z) \equiv 0$ the extremal points were introduced by Fekete [5]. In the case that $E \subset C^{\prime}$ and $b(z)$ is bounded, extremal points were introduced by Leja [11] and investigated later by him and his students in connection with the Dirichlet boundary value problem and conformal mapping of simple and multiconnected domains on some canonical domains (for bibliography see [14]). 
Using the same procedure, one may introduce extremal points of $E$ connected with homogeneous polynomials. If $b(z)$ is lower semicontinuous, there exists a system

$$
h^{(v)}=\left\{h_{1}^{(v)}, h_{2}^{(v)}, \ldots, h_{v_{0}}^{(v)}\right\}, \quad v_{0}=C_{v+n-1, n-1},
$$

of points of $E$ such that

where

$$
\left|W\left(h^{(v)}, b\right)\right|=\max _{p^{(v)} \subset E}\left|W\left(p^{(v)}, b\right)\right|, \quad p^{(v)}=\left\{p_{1}, \ldots, p_{v_{0}}\right\}
$$

$$
W\left(p^{(v)}, b\right)=W\left(p^{(v)}\right) \exp \left[-v \sum_{i=1}^{v_{0}} b\left(p_{l}\right)\right], \quad v=0,1,2, \ldots .
$$

In the case of $n=2$ the extremal points (5) have been introduced by Leja [9] $(b(z) \equiv 0)$ and applied by him to the investigation of domains of uniform convergency of the series of homogeneous polynomials of two complex variables (see $[9 ; 12])$. Exploitation of the points $(4)(b \equiv 0)$ to the same purpose in the case of $C^{n}, n \geqq 3$, has been done in [19].

Let us define $v_{v}(E, b)$ and $w_{v}(E, b)$ by

$$
\begin{aligned}
& v_{v}(E, b)=\left[\left|V\left(q^{(v)}, b\right)\right|\right]^{1 / n C_{v+n, n+1}}, v=1,2, \ldots, \\
& w_{v}(E, b)=\left[\left|W\left(h^{(v)}, b\right)\right|\right]^{1 / v C_{v+n-1, n-1}}, \quad v=1,2, \ldots .
\end{aligned}
$$

One can prove that the numbers $v_{v}(E, 0)$ and $w_{v}(E, 0)$ are invariant with respect to the unitary transformations of $C^{n}$ onto itself.

It is known $[9 ; 10 ; 11 ; 14]$ that the sequence $\left\{v_{v}(E, b)\right\}, E \subset C^{1}$, and the sequence $\left\{w_{v}(E, b)\right\}, E \subset C^{2}$, are both convergent. Convergence of $\left\{w_{v}(E, b)\right\}$ for $E \subset C^{1}$ is trivial. The limit $v(E, 0)=\lim v_{v}(E, 0)$ is called the transfinite diameter of $E$ (= logarithmic capacity of $E$ ). The limit $w(E, 0)=\lim w_{v}(E, 0), E \subset C^{2}$, is a triangular transfinite diameter of $E[9 ; 12]$. The question (formulated by Leja [16] in a slightly different form) as to whether the sequences (7) or (8) for $E \subset C^{n}, n \geqq 2$, are convergent or not remains still unsolved (except for $E=E_{1}$ $\left.\times E_{2} \times \ldots \times E_{n}\right)$.

Remark on Šlov's boundary. Let $S(E)$ denote Silov's boundary of $E$ with respect to polynomials and let $E^{*}$ denote the topological sum of all ordinary extremal points of $E$ with respect to $b(z) \equiv 0$. The extremal points of $q^{(v)}$ are not unique in general. Therefore, $E^{*}$ may a priori depend on which extremal points of order $v$ we choose for $v=1,2, \ldots$. There is, of course, at least one $E^{*}$ such that $E^{*} \subset S(E)$. But, as we shall see from Lemma 1 , $\S 6$, any polynomial takes its maximum on $E^{*}$. Therefore, we always have $S(E) \subset E^{*}$. We know [18] that in the case of $C^{1}$ the set $E^{*}$ is unique and therefore $E^{*}=S(E)$. The author can prove that $E^{*}$ is unique also if $E \subset C^{n}, n=2$, is circular. However, we do not know what is the answer in the general case. 
6. The extremal function $\Phi(z, E, b)$. Let $E$ be a bounded closed unisolvent set in $C^{n}$. Given an arbitrary real function $b(z)$ defined and bounded on $E$ and an arbitrary unisolvent system

$$
p^{(v)}=\left\{p_{1}, \ldots, p_{v_{*}}\right\}, \quad v=1,2, \ldots, v_{*}=C_{v+n, n},
$$

of points of $E$, the functions

$$
\Phi^{(i)}\left(z, p^{(v)}, b\right)=L^{(i)}\left(z, p^{(v)}\right) e^{v b\left(p_{i}\right)}, \quad i=1,2, \ldots, v_{*},
$$

where $L^{(i)}\left(z, p^{(v)}\right)$ denote the polynomials (2.6), are polynomials of degree $v$ such that

$$
\Phi^{(i)}\left(p_{j}, p^{(v)}, b\right)=\delta_{i j} e^{v b\left(p_{i}\right)}, \quad i, j=1,2, \ldots, v_{*}
$$

For any $v=1,2, \ldots$ we define extremal functions $\Phi_{v}^{(i)}(z, E, b), i=1,2,3,4$, corresponding to $E$ and $b$, by the formulas

$$
\begin{aligned}
& \Phi_{v}^{(1)}(z, E, b)=\max _{1 \leqq i \leqq v_{*}}\left|\Phi^{(i)}\left(z, \gamma^{(v)}, b\right)\right|, \\
& \Phi_{v}^{(2)}(z, E, b)=\sum_{i=0}^{v *}\left|\Phi^{(i)}\left(z, \gamma^{(v)}, b\right)\right|, \\
& \Phi_{v}^{(3)}(z, E, b)=\inf _{p^{(v)} \subset E}\left\{\max _{(i)}\left|\Phi^{(i)}\left(z, p^{(v)}, b\right)\right|\right\}, \\
& \Phi_{v}^{(4)}(z, E, b)=\inf _{p^{(v)} \subset E} \sum_{i=1}^{v_{i}}\left|\Phi^{(i)}\left(z, p^{(v)}, b\right)\right|,
\end{aligned}
$$

where $\gamma^{(v)}=\left\{\gamma_{1}^{(v)}, \ldots, \gamma_{v *}^{(v)}\right\}$ denotes extremal system (5.3).

THEOREM 1. The sequences $\left\{\left[\Phi_{v}^{(i)}(z, E, b)\right]^{1 / v}\right\}, i=1,2,3,4$, are convergent at any point $z \in C^{n}$ to the same limit $\Phi(z, E, b)$,

$$
\Phi(z, E, b)=\lim _{v \rightarrow \infty}\left[\Phi_{v}^{(i)}(z, E, b)\right]^{1 / v}, \quad z \in C^{n}, \quad i=1,2,3,4,
$$

(the limit $\Phi(z, E, b)$ being finite or not).

Proof. $1^{\circ}$ First of all we shall prove that the sequence $\left\{\left(\Phi_{(v)}^{(1)}\right)^{1 / v}\right\}$ has a limit (finite or not) at any point $z \in C^{n}$. Due to (2.6) and (5.1) we have

$$
\Phi^{(i)}\left(z, \gamma^{(v)}, b\right)=\frac{V_{i}\left(z, \gamma^{(v)}, b\right)}{V\left(\gamma^{(\nu)}, b\right)}, \quad i=1,2 \ldots, v_{*},
$$

where $V_{i}\left(z, \gamma^{(v)}, b\right)$ is a determinant $(5.1)$ corresponding to $\left\{\gamma_{1}^{(v)}, \gamma_{2}^{(v)}, \ldots, \gamma_{i-1}^{(v)}, z\right.$, $\left.\gamma_{i+1}^{(v)}, \ldots, \gamma_{v_{*}}^{(v)}\right\}$. Therefore, in virtue of (5.4)

$$
\left|\Phi^{(i)}\left(z, \gamma^{(v)}, b\right)\right|<\alpha_{v} \exp [v b(z)], \quad z \in E, \quad i=1,2, \ldots, v_{*} .
$$

Let $z$ be an arbitrary fixed point of $C^{n}$, let $v$ be an arbitrary fixed positive integer and let $\mu$ be an arbitrary integer greater or equal to $v$. There exist two 
uniquely determined integers $k$ and $r$ such that $\mu=k v+r$ and $0 \leqq r<v$. By the interpolation formula (2.8) and due to inequality (7) we have

$$
\left|\left[\Phi^{(i)}\left(z, \gamma^{(v)}, b\right)\right]^{k}\right| \leqq \alpha_{v}^{k} \sum_{j=1}^{\mu_{*}} \exp \left[k v b\left(\gamma_{j}^{(\mu)}\right)\right]\left|L^{(j)}\left(z, \gamma^{(\mu)}\right)\right|, \quad i=1,2, \ldots, v_{*} .
$$

Let $b_{0}=\inf _{z \in E} b(z)$. Then

$$
\exp \left[k v b\left(\gamma_{j}\right)\right]=\exp \left[(\mu-r) b\left(\gamma_{j}\right)\right] \leqq \exp \left[\mu b\left(\gamma_{j}\right)-r b_{0}\right]
$$

Therefore,

$\left|\Phi^{(i)}\left(z, \gamma^{(v)}, b\right)\right|^{k} \leqq \alpha_{\nu}^{k} \exp \left[-r b_{0}\right] \sum_{j=1}^{\mu_{*}}\left|\Phi^{(j)}\left(z, \gamma^{(\mu)}, b\right)\right| \leqq \mu_{*} \alpha_{v}^{k} \exp \left[-r b_{0}\right] \Phi_{\mu}^{(1)}(z, E, b)$,

whence

$$
\left[\left(\Phi_{v}^{(1)}(z, E, b)\right)^{1 / v}\right]^{v k / \mu} \leqq\left(\alpha_{v}\right)^{1 / v}\left[\mu_{*} e^{-r b_{0}}\right]^{1 / \mu}\left[\Phi_{\mu}^{(1)}(z, E, b)\right]^{1 / \mu}, \quad \mu=1,2, \ldots .
$$

Since $v k / \mu \rightarrow 1$ and $\left(\mu_{*} \exp \left(-r b_{0}\right)\right)^{1 / \mu} \rightarrow 1$, as $\mu \rightarrow \infty$, we have

$$
\left[\Phi_{v}^{(1)}(z, E, b)\right]^{1 / v} \leqq\left(\alpha_{v}\right)^{1 / v} \liminf _{\mu \rightarrow \infty}\left[\Phi_{\mu}^{(1)}(z, E, b)\right]^{1 / \mu}, \quad v=1,2, \ldots,
$$

whence due to (5.2)

$$
\limsup _{v \rightarrow \infty}\left[\Phi_{\nu}^{(1)}(z, E, b)\right]^{1 / v} \leqq \lim _{\mu \rightarrow \infty}\left[\Phi_{\mu}^{(1)}(z, E, b)\right]^{1 / \mu}, \quad z \in C^{n} .
$$

Therefore, the sequence $\left\{\left(\Phi_{v}^{(1)}\right)^{1 / v}\right\}$ has the limit $\Phi(z)=\Phi(z, E, b)$ (finite or not).

$2^{\circ}$ To prove that the sequences $\left\{\left[\Phi^{(i)}(z, E, b)\right]^{1 / v}\right\}, i=2,3,4$, are convergent to $\Phi(z)$ it is sufficient to show that

$$
\Phi_{v}^{(1)} \leqq \alpha_{v} \Phi_{v}^{(4)} \leqq \alpha_{v} \Phi_{v}^{(2)} \leqq\left(v_{*}\right)^{2} \alpha_{v}^{2} \Phi_{v}^{(3)} \leqq\left(v_{*}\right)^{2} \alpha_{v}^{2} \Phi_{v}^{(1)}, \quad v=1,2, \ldots
$$

By the interpolation formula (2.8) and due to (7) we have

$$
\left|\Phi^{(i)}\left(z, \gamma^{(v)}, b\right)\right| \leqq \alpha_{v} \sum_{j=1}^{v_{*}}\left|\Phi^{(j)}\left(z, p^{(v)}, b\right)\right|, \quad z \in C^{n}, \quad i=1,2, \ldots, v_{*},
$$

$p^{(v)}$ being an arbitrary unisolvent system in $E$ of order $v$. Thus $\Phi_{v}^{(1)} \leqq \alpha_{v} \Phi_{v}^{(4)}$. The inequality $\Phi_{v}^{(4)} \leqq \Phi_{v}^{(2)}$ follows directly from (4) and (6). Further, it follows from (10) that

$$
\sum_{i=1}^{v_{*}}\left|\Phi^{(i)}\left(z, \gamma^{(v)}, b\right)\right| \leqq v_{*}^{2} \alpha_{v} \inf _{p^{(v)} \subset E}\left\{\max _{(j)}\left|\Phi^{(j)}\left(z, p^{(v)}, b\right)\right|\right\},
$$

whence $\Phi_{v}^{(2)} \leqq v_{*}^{2} \alpha_{v} \Phi_{v}^{(3)}$. Finally, the inequality $\Phi_{v}^{(3)} \leqq \Phi_{v}^{(1)}$ follows directly from (3) and (5). The proof is completed.

LEMMA 1. If $P_{v}(z)$ denotes an arbitrary polynomial of degree $v$ such that

$$
\left|P_{v}(z)\right| \leqq M e^{v b(z)}, \quad z \in E, \quad M=\text { const, }
$$

then

$$
\left|P_{v}(z)\right| \leqq M \Phi^{v}(z, E, b), \quad z \in C^{n}
$$


Proof. By the interpolation formula (2.8) and due to (11) we have

$$
\left|\left[P_{v}(z)\right]^{\mu}\right| \leqq M^{\mu} \sum_{i=1}^{(\mu \nu) *}\left|\Phi^{(i)}\left(z, \gamma^{(\mu v)}, b\right)\right|=M^{\mu} \Phi_{\mu \nu}^{(2)}, \quad \mu=1,2, \ldots
$$

Therefore, $\left|P_{v}(z)\right| \leqq M\left[\Phi_{\mu \nu}^{(2)}(z, E, b)\right]^{1 / \mu}, \mu=1,2, \ldots$, whence (12) follows.

Let $D(E)$ denote the unbounded component of $C E$. We know $[10 ; 7]$ that if $E \subset C^{1}$ is of positive logarithmic capacity and $b(z) \equiv 0$, then $\Phi(z, E, 0) \equiv$ $\exp G(z, E)$, where $G(z, E)$ is a Green's function of $D(E)$ with pole at $\infty$. Therefore, (12) is a generalization of the Bernstein-Walsh inequality [27, p. 77].

THEOREM 2. Let $A_{v}(E, b)$ denote the family of all polynomials of degree $v$ such that $\left|P_{v}(z)\right| \leqq M_{v} \exp [v b(z)], z \in E, M_{v}=$ const, $\left(M_{v}\right)^{1 / v} \rightarrow 1$. Then

$$
\Phi(z, E, b)=\lim _{v \rightarrow \infty}\left\{\sup _{P_{v} \in A_{v}}\left(\left|P_{v}(z)\right|\right)^{1 / v}\right\}, \quad z \in C^{n} .
$$

Proof. By Lemma 1 we have

$$
\left|P_{v}(z)\right| \leqq M_{v} \Phi^{v}(z, E, b), \quad z \in C^{n}, \quad v=1,2, \ldots .
$$

On the other hand, by (7) the polynomials $\alpha_{v}^{-1} M_{v} \Phi^{(i)}\left(z, \gamma^{(v)}, b\right), i=1,2, \ldots, v_{*}$, belong to $A_{v}(E, b), v=1,2, \ldots$. Therefore (13) is true.

REMARK. Let

$$
R_{v}(z, E, b)=\sup _{P_{v} \in A_{v}}\left|\frac{P_{v}(z)}{M_{v}}\right|, \quad v=1,2 \ldots
$$

Then $R_{\mu+v}(z) \geqq R_{\mu}(z) R_{v}(z), \mu, v=1,2, \ldots$, whence it follows that there exists the limit $R(z)=\lim _{v \rightarrow \infty}\left[R_{v}(z, E, b)\right]^{1 / v}, z \in C^{n}$.

7. Some fundamental properties of $\Phi(z, E, b)$. Let $b_{0}=\inf _{z \in E} b(z)$ and $B_{0}$ $=\sup _{z \in E} b(z)$ Then

$$
e^{b_{0}} \leqq e^{b_{0}} \Phi(z, E, 0) \leqq \Phi(z, E, b) \leqq e^{B_{0}} \Phi(z, E, 0), \quad z \in C^{n} .
$$

Indeed, since $\left|\Phi^{(i)}\left(z, \gamma^{(v)}, b\right)\right| \geqq\left|L^{(i)}\left(z, \gamma^{(v)}\right)\right| e^{v b_{0}}, i=1,2, \ldots, v_{*}$, then $\Phi_{v}^{(1)}(z, E, b)$ $\geqq \Phi_{v}^{(3)}(z, E, 0) e^{v b_{0}}$, whence $\Phi(z, E, b) \geqq \Phi(z, E, 0) e^{b_{0}}$. On the other hand,

$$
\sum_{i=1}^{v_{*}} L^{(i)}\left(z, \gamma^{(v)}\right) \equiv 1, z \in C^{n},
$$

therefore, $\Phi_{v}^{(1)}(z, E, 0) \geqq 1 / v_{*}$, whence

$$
\Phi(z, E, 0) \geqq 1, \quad z \in C^{n} .
$$

Thus we proved the first two inequalities in (1).

Since for any unisolvent system $p^{(v)} \subset E$ we have

$$
\left|\Phi^{(i)}\left(z, p^{(v)}, b\right)\right|<\exp \left(v B_{0}\right)\left|L^{(i)}\left(z, p^{(v)}\right)\right|,
$$

then $\Phi_{v}^{(4)}(z, E, b) \leqq \exp \left(v B_{0}\right) \Phi_{v}^{(4)}(z, E, 0)$, whence the last inequality of (1) follows.

The complement $C E$ of $E$ in the space $C^{n}$ consists of at most a countable num- 
ber of disjoint domains $C E=\left\{D_{v}\right\}+D_{\infty}$, where $D_{\infty}=D(E)$ is unbounded. By the maximum modulus principle for holomorphic functions the ordinary extremal points of $E$ with respect to $b(z) \equiv 0$ lie on the boundary of $D_{\infty}$. Therefore the extremal systems of $E$ and $\Delta=U \bar{D}_{v}$ with respect to $b(z) \equiv 0$ are the same. Thus,

$$
\Phi(z, E, 0) \equiv \Phi(z, \Delta, 0), \quad z \in C^{n} .
$$

As a simple consequence of (6.7), (2) and (3) we obtain

$$
\Phi(z, E, 0)=1 \quad \text { for } z \in \Delta .
$$

The following three properties follow directly from the definition (6.6) of $\Phi_{v}^{(4)}$ and from Theorem 1, $\$ 6$ :

$$
\begin{gathered}
\Phi\left(z, E, b_{1}\right)=e^{c} \Phi(z, E, b), \quad z \in C^{n}, \quad b_{1}(z)=b(z)+c, \quad c=\text { const; } \\
\Phi(z, E, b) \leqq \Phi(z, F, b),
\end{gathered}
$$

$z \in C^{n}$, if $F \subset E$ is a unisolvent closed subset of $E$; and

$$
\Phi\left(z, E, b_{1}\right) \leqq \Phi\left(z, E, b_{2}\right),
$$

$z \in C^{n}$, if $b_{1}(z) \leqq b_{2}(z)$ for $z \in E$.

Now we shall prove a less obvious property of $\Phi$, namely,

$$
\prod_{i=1}^{s} \Phi\left(z, E, b_{i}\right) \leqq \Phi^{s}(z, E, b), \quad z \in C^{n}, \quad b(z)=\frac{1}{s}\left[b_{1}(z)+\ldots+b_{s}(z)\right] .
$$

We shall prove (8) for $s=2$. If $s>2$, the proof is quite analogous. Let $b(z)$ $=\left[b_{1}(z)+b_{2}(z)\right] / 2$ and let $\gamma^{(v, i)}=\left\{\gamma^{(v, i)}, \ldots, \gamma_{v_{*}}^{(v, i)}\right\}, i=1,2$, be the $v$ th extremal system of $E$ with respect to $b_{i}(z), i=1,2$, respectively. Let

$$
\gamma^{(2 v)}=\left\{\gamma_{1}^{(2 v)}, \gamma_{2}^{(2 v)}, \ldots, \gamma_{(2 v)}^{(2 v)}\right\}
$$

be the (2v)th extremal system of $E$ with respect to $b(z)$. Given $z_{0} \in C^{n}$, there are integers $i_{1}$ and $i_{2}$ such that

$$
\left|\Phi^{\left(i_{k}\right)}\left(z_{0}, \gamma^{(v, k)}, b_{k}\right)\right|=\Phi_{v}^{(1)}\left(z_{0}, E, b_{k}\right), \quad k=1,2 .
$$

Due to the interpolation formula (2.8) and by (6.7) we have

Therefore,

$$
\begin{gathered}
\left|\Phi^{\left(i_{1}\right)}\left(z, \gamma^{(v, 1)}, b_{1}\right) \Phi^{\left(i_{2}\right)}\left(z, \gamma^{(v, 2)}, b_{2}\right)\right| \\
\leqq \alpha_{v}^{2} \sum_{i=1}^{(2 v) * *} \exp \left(2 v \frac{b_{1}\left(\gamma_{i}^{(2 v)}\right)+b_{2}\left(\gamma_{i}^{(2 v)}\right)}{2}\left|L^{(i)}\left(z, \gamma^{(2 v)}\right)\right| .\right)
\end{gathered}
$$

$$
\Phi_{v}^{(1)}\left(z_{0}, E, b_{1}\right) \Phi_{v}^{(1)}\left(z_{0}, E, b_{2}\right) \leqq \alpha_{v}^{2} \Phi_{2 v}^{(2)}\left(z_{0}, E, b\right), \quad v=1,2, \ldots,
$$

whence (10) follows.

Due to (6.8) and Theorem 1, §6, we have

$$
\left[\Phi_{v}^{(1)}(z, E, b)\right]^{1 / v} \leqq \Phi(z, E, b), \quad z \in C^{n}, \quad v=1,2, \ldots .
$$


Thus $\Phi$ is an upper bound of continuous functions $\left(\Phi_{v}^{(1)}\right)^{1 / v}, v=1,2, \ldots$ Therefore, $\Phi(z, E, b)$ is lower semicontinuous in $C^{n}$. Function $(1 / v) \log \left|\Phi^{(i)}\left(z, \gamma^{(v)}, b\right)\right|$, $i=1,2, \ldots, v_{*}$, is plurisubharmonic in $C^{n}$, so the function $(1 / v) \log \Phi_{v}^{(i)}(z, E, b)$ $=\max _{i}(1 / v) \log \left|\Phi^{(i)}\left(z, \gamma^{(v)}, b\right)\right|$ is also plurisubharmonic in $C^{n}$. Since $\Phi(z, E, b)$ is an upper limit of plurisubharmonic functions $\left[\Phi_{v}^{(1)}(z, E, b)\right]^{1 / \nu}$, the function

$$
\log \Phi^{*}(z, E, b)=\lim \sup _{z^{\prime} \rightarrow z} \log \Phi\left(z^{\prime}, E, b\right), \quad z, z^{\prime} \in C^{n}
$$

is also plurisubharmonic in any domain $D \subset C^{n}$ in which $\Phi(z, E, b)$ is bounded.

COROLlaRY 1. If $E$ is a Šlov boundary (with respect to polynomials) of a bounded domain $D$, then $\log \Phi^{*}(z, E, b)$ is plurisubharmonic in $D$.

Let $\mathscr{P}(E)$ denote a set of the points $z_{0} \in C^{n}$ such that for any polynomial $P(z)$ we have

We claim that

$$
\left|P\left(z_{0}\right)\right| \leqq \max _{z \in E}|P(z)|
$$

$$
\mathscr{P}(E)=\{z \mid \Phi(z, E, 0)=1\} .
$$

At first we shall prove that if $z_{0} \in \mathscr{P}(E)$, then $\Phi\left(z_{0}, E, 0\right)=1$. If this was not true then by (2) we would have $\Phi\left(z_{0}, E, 0\right)>1$. Therefore, there would exist integers $v$ and $i_{0}, 1 \leqq i_{0} \leqq v_{*}$, such that $\left|L^{\left(i_{0}\right)}\left(z_{0}, \gamma^{(v)}\right)\right|>1$. But $L^{\left(i_{0}\right)}\left(z, \gamma^{(v)}\right)$ is a polynomial of degree $v$ and $\left|L^{\left(i_{0}\right)}\left(z, \gamma^{(v)}\right)\right| \leqq 1$ for $z \in E$, whence by the definition of $\mathscr{P}(E)$ we would have $\left|L^{\left(i_{0}\right)}\left(z_{0}, \gamma^{(v)}\right)\right| \leqq 1$. We have obtained a contradiction. Thus $\Phi\left(z_{0}, E, 0\right)=1$. On the other hand, if $\Phi\left(z_{0}, E, 0\right)=1$ and $P(z)$ is an arbitrary polynomial of degree $v$, then due to (6.12) we have

$$
\left|P\left(z_{0}\right)\right| \leqq\left(\max _{z \in E}|P(z)|\right) \Phi^{y}\left(z_{0}, E, 0\right)=\max _{z \in E}|P(z)| .
$$

Therefore, if $\Phi\left(z_{0}, E, 0\right)=1$ then $z_{0} \in \mathscr{P}(E)$. The proof is completed.

8. The function $\Phi(z, E, 0)$ for $E=E_{1} \times E_{2} \times \ldots \times E_{n}$. The following lemma has been proved in [15].

LEMMA 1. If $E$ is a compact plane set of positive logarithmic capacity, then there exist points $x_{v} \in E, v=0,1, \ldots$, such that $x_{i} \neq x_{j}$ for $i \neq j$ and

$$
\left|\omega_{v}(z)\right| \leqq\left|\omega_{v}\left(x_{v}\right)\right|, \quad z \in E, \quad v=1,2, \ldots,
$$

where

$$
\omega_{v}(z)=\left(z-x_{0}\right)\left(z-x_{1}\right) \ldots\left(z-x_{v-1}\right), \quad v=1,2, \ldots .
$$

Moreover, the sequence $\left\{\left(\left|\omega_{v}(z) / \omega_{v}\left(x_{v}\right)\right|\right)^{1 / v}\right\}$ converges uniformly to $\Phi(z, E, 0)$ on any closed subset of $C E$.

Now we shall prove

Lemma 2. Let $E=E_{1} \times \ldots \times E_{n}$, where $E_{k}$ is a compact set of positive logarithmic capacity in the complex $z_{k}$-plane. If $P_{v}(z)=P_{v}\left(z_{1}, \ldots, z_{n}\right)$ is a polynomial of degree $v$ such that 


$$
\left|P_{v}(z)\right| \leqq M \text { for } z \in E \text {, }
$$

then

$$
\left|P_{v}(z)\right| \leqq M \max \left\{\Phi^{v}\left(z_{1}, E_{1}, 0\right), \ldots, \Phi^{v}\left(z_{n}, E_{n}, 0\right)\right\}, \quad z \in C^{n} .
$$

Proof. Let

$$
x_{k 0}, x_{k 1}, \ldots, x_{k v}, \ldots
$$

denote the sequence of points of $E_{k}$ whose existence is assured by Lemma 1 . Let

$$
\omega_{k v}\left(z_{k}\right)=\left(z_{k}-x_{k 0}\right) \ldots\left(z_{k}-x_{k v-1}\right), \quad v=1,2, \ldots, \quad k=1,2, \ldots, n .
$$

Then

$$
\left|\frac{\omega_{k v}\left(z_{k}\right)}{\omega_{k v}\left(x_{k v}\right)}\right| \leqq 1, \quad z_{k} \in E_{k}, \quad v=1,2, \ldots,
$$

and the sequence $\left\{\left(\left|\omega_{k v}\left(z_{k}\right) / \omega_{k v}\left(x_{k v}\right)\right|\right)^{1 / v}\right\}$ converges uniformly to $\Phi\left(z_{k}, E_{k}, 0\right)$ on any closed subset of $C E_{k}$. By Newton's interpolation formula we have

$$
P_{v}^{\mu}(z) \equiv \sum_{i_{1}+\ldots+i_{n} \leqq \mu v} a_{i_{1} i_{2} \ldots i_{n}} \prod_{k=1}^{n} \omega_{k i_{k}}\left(z_{k}\right), \quad\left(\omega_{k 0}\left(z_{k}\right) \equiv^{d f} 1\right)
$$

where

$$
a_{i_{1} \ldots i_{n}}=\frac{1}{(2 \pi i)^{n}} \int_{C_{1}} \ldots \int_{C_{n}} \frac{P_{v}^{\mu}\left(\zeta_{1}, \ldots, \zeta_{n}\right) d \zeta_{1} \ldots d \zeta_{n}}{\prod_{k=1}^{n} \omega_{k i_{k}+1}\left(\zeta_{k}\right)},
$$

$C_{1}, \ldots, C_{n}$ being smooth suitably oriented curves, which contain in their interiors $E_{1}, E_{2}, \ldots, E_{n}$, respectively. Since $E_{k}$ may be approximated from the outside by regular sets (for which the function $\Phi$ is continuous) and the function $\Phi\left(z_{k}, E_{k}, 0\right)$ is continuous with respect to the sets, it is sufficient to prove our lemma only for regular sets. Assuming $E_{k}$ to be regular and $\varepsilon$ to be an arbitrary positive number, let $R>1$ be so near to 1 that $R-\varepsilon<1$ and

where

$$
\left|P_{v}(z)\right|<(1+\varepsilon) M \text { for } z \in C=C_{1} \times C_{2} \times \ldots \times C_{n},
$$

$$
C_{k}=\left\{z_{k} \mid \Phi\left(z_{k}, E_{k}, 0\right)=R\right\}, \quad k=1,2, \ldots, n .
$$

For these $C_{k}$ we have by (3) and (7)

$$
\left|a_{i_{1} \ldots i_{n}}\right| \leqq(1+\varepsilon)^{\mu} M^{\mu} M_{1} / \prod_{k=1}^{n} \min _{\zeta_{k} \in C_{k}}\left|\omega_{k i_{k}}\left(\zeta_{k}\right)\right|,
$$

$M_{1}$ being a constant which depends only on $C$. Therefore,

$$
\left|P_{v}^{\mu}(z)\right| \leqq(1+\varepsilon)^{\mu} M^{\mu} M_{1} \sum_{i_{1}+\ldots+i_{n} \leqq u v} \prod_{k=1}^{n}\left|\frac{\omega_{k i_{k}}\left(z_{k}\right)}{\omega_{k i_{k}}\left(x_{k i_{k}}\right)}\right| \prod_{k=1}^{n} \max _{\zeta_{k} \in C_{k}}\left|\frac{\omega_{k i_{k}}\left(x_{k i_{k}}\right)}{\omega_{k i_{k}}\left(\zeta_{k}\right)}\right| .
$$

It follows from (5) and (6.12) that $\left|\omega_{k i_{k}}\left(z_{k}\right) / \omega_{k i_{k}}\left(x_{k i_{k}}\right)\right| \leqq \Phi^{i_{k}}\left(z_{k}, E_{k}, 0\right), z_{k}$ being arbitrary. By Lemma 1 there is an $l_{0}>0$ such that

$$
\left|\omega_{k l}\left(z_{k}\right) / \omega_{k l}\left(x_{k l}\right)\right| \geqq(R-\varepsilon)^{l} \text { for } z_{k} \in C_{k}, \quad k=1,2 \ldots, n, l \geqq l_{0} .
$$


On the other hand, since $C_{k} \cap E_{k}=\varnothing$ and $C_{k}$ is bounded, there is a constant $\delta<1$ such that

$$
\left|\omega_{k l}\left(z_{k}\right) / \omega_{k l}\left(x_{k l}\right)\right| \geqq \delta(R-\varepsilon)^{l}, \quad z_{k} \in C_{k}, \quad k=1,2 \ldots, n, l=1,2, \ldots, l_{0} .
$$

Therefore,

$$
\left|P_{v}^{\mu}(z)\right| \leqq(1+\varepsilon)^{\mu} M^{\mu} M_{1} \max _{1 \leqq k \leqq n}\left\{\Phi^{\mu v}\left(z_{k}, E_{k}, 0\right)\right\} \times \sum_{i_{1}+\ldots+i_{n} \leqq \mu \nu} \frac{1}{\delta^{n}(R-\varepsilon)^{i_{1}+\cdots+i_{n}}}
$$

whence

$$
\left|P_{v}^{\mu}(z)\right| \leqq(1+\varepsilon)^{\mu} M^{\mu} M_{1} \delta^{-n C_{\mu \nu+n, n}}(R-\varepsilon)^{-\mu \nu} \max _{1 \leqq k \leqq n}\left\{\Phi^{\mu \nu}\left(z_{k}, E_{k}, 0\right)\right\}, \quad z \in C^{n} .
$$

After taking the $\mu$ th root of both sides of this inequality and letting $\mu$ go to $\infty$, we shall find

$$
\left|P_{v}(z)\right| \leqq \frac{1+\varepsilon}{(R-\varepsilon)^{\nu}} M \max _{1 \leqq k \leqq n}\left\{\Phi^{v}\left(z_{k}, E_{k}, 0\right)\right\}, \quad z \in C^{n} .
$$

Since $\varepsilon>0$ may be arbitrarily small and $R$ may be arbitrarily close to 1 , we conclude that inequality (4) holds.

THEOREM 1. If $E=E_{1} \times \ldots \times E_{n}$, then

$$
\Phi(z, E, 0)=\max _{1 \leqq k \leqq n}\left\{\Phi\left(z_{k}, E_{k}, 0\right)\right\}, \quad z \in C^{n} .
$$

Proof. If $P_{v}\left(z_{k}\right)$ is a polynomial in $z_{k}$ of degree $v$, then it is a polynomial of the same degree in $z=\left(z_{1}, \ldots, z_{n}\right)$, whence by $(6.12)$

$$
\left|P_{v}\left(z_{k}\right)\right| \leqq\left(\max _{z \in E}\left|P_{v}\left(z_{k}\right)\right|\right) \phi^{v}(z, E, 0), \quad z \in C^{n} .
$$

Therefore due to Theorem 2, §6,

$$
\Phi\left(z_{k}, E_{k}, 0\right) \leqq \Phi(z, E, 0), \quad k=1,2, \ldots, n .
$$

On the other hand, by Lemma 2 and in virtue of Theorem 2, $\$$, we have

$$
\Phi(z, E, 0) \leqq \max _{1 \leqq k \leqq n}\left\{\Phi\left(z_{k}, E_{k}, 0\right)\right\}, \quad z \in C^{n} .
$$

Now (8) follows immediately from (9) and (10).

Corollary 1. If $d\left(E_{k}\right)>0, k=1,2, \ldots, n$, then $\log \Phi^{*}(z, E, 0)$, where $E=E_{1}$ $\times \ldots \times E_{n}$, is plurisubharmonic in $C^{n}$.

Corollary 2. If $E_{k}, k=1,2, \ldots, n$, is regular and $E=E_{1} \times \ldots \times E_{n}$, then $\Phi(z, E, 0)$ is continuous in $C^{n}$.

REMARK. It follows from (8) that if $E_{k_{0}}$ for some $1 \leqq k_{0} \leqq n$ is not regular, then $\Phi(z, E, 0), E=E_{1} \times \ldots \times E_{n}$, is discontinuous at some points outside of $E$, e.g., if $\Phi\left(z_{1}, E_{1}, 0\right)$ is not continuous at a point $z_{1}^{0} \in E_{1}$, then the function $\Phi(z, E, 0)$ is discontinuous at any point $\left(z_{1}^{0}, z_{2}, \ldots, z_{n}\right)$, where $\left(z_{2}, \ldots, z_{n}\right) \notin E_{2} \times$ $\ldots \times E_{n}$.

9. The function $\Phi(z, E, 0)$ for circular sets. The set $E \subset C^{n}$ is called circular if along with the point $z^{0}=\left(z_{1}^{0}, \ldots, z_{n}^{0}\right) \in E$ all the points of the circle 


$$
z=e^{i \theta} z^{0} \equiv\left(e^{i \theta} z_{1}^{0}, \ldots, e^{i \theta} z_{n}^{0}\right), \quad 0 \leqq \theta \leqq 2 \pi,
$$

belong to $E$.

The set $E \subset C^{n}$ is called a Reinhardt circular set if along with the point $z^{0}$ $=\left(z_{1}^{0}, \ldots, z^{0}\right) \in E$ also the set

belongs to $E$.

$$
\left\{z|| z_{k}|=| z_{k}^{0} \mid, \quad k=1,2, \ldots, n\right\}
$$

Let $E$ be a bounded closed subset of $C^{n}$, unisolvent with respect to homogeneous polynomials. The function $b(z)$ being defined and lower semicontinuous on $E$, let

$$
h^{(v)}=\left\{h_{1}^{(v)}, \ldots, h_{v}^{(v)}\right\}, \quad v_{0}=C_{v+n-1, n-1}
$$

be the vth extremal system of $E$ defined by (5.5) and (5.6). If $p^{(v)}=\left\{p_{1}, \ldots, p_{v_{0}}\right\}$ is an arbitrary unisolvent system of points of $E$, then the functions

$$
\psi^{(i)}\left(z, p^{(v)}, b\right)=T^{(i)}\left(z, p^{(v)}\right) e^{v b\left(p_{i}\right)}, \quad i=1,2, \ldots, v_{0},
$$

$T^{(i)}\left(z, p^{(v)}\right)$ denoting the polynomial (2.12), are homogeneous polynomials of degree $v$. Define the extremal functions $\psi_{v}^{(i)}(z, E, b), i=1,2,3,4$, corresponding to $E$ and $b$ by the formulas

$$
\begin{aligned}
& \psi_{v}^{(1)}(z, E, b)=\max _{(i)}\left|\psi^{(i)}\left(z, h^{(v)}, b\right)\right|, \quad z \in C^{n}, \\
& \psi_{v}^{(2)}(z, E, b)=\sum_{i=1}^{v_{0}}\left|\psi^{(i)}\left(z, h^{(v)}, b\right)\right|, \quad z \in C^{n}, \\
& \psi_{v}^{(3)}(z, E, b)=\inf _{p^{(v)} \subset E}\left\{\max _{(i)}\left|\psi^{(i)}\left(z, p^{(v)}, b\right)\right|\right\}, \quad z \in C^{n}, \\
& \psi_{v}^{(4)}(z, E, b)=\inf _{p^{(v)} \subset E}\left\{\sum_{i=1}^{v_{0}}\left|\psi^{(i)}\left(z, p^{(v)}, b\right)\right|\right\}, \quad z \in C^{n} .
\end{aligned}
$$

By reasoning quite analogous to the reasoning of $\$ 6$ we may prove (see also [19])

THEOREM 1. At any point $z \in C^{n}$ the sequences $\left\{\left(\psi_{\nu}^{(i)}(z, E, b)^{1 / v}\right\}, i=1,2,3,4\right.$, are convergent to the same limit $\psi(z, E, b)$,

$$
\psi(z, E, b)=\lim _{v \rightarrow \infty}\left(\psi^{(i)}(z, E, b)\right)^{1 / v}, \quad z \in C^{n}, \quad i=1,2,3,4 .
$$

Lemma 1 of $\$ 6$ now takes the form of

LEMMA 1. If $Q_{v}(z)$ denotes an arbitrary homogeneous polynomial of degree $v$ such that

then

$$
\left|Q_{v}(z)\right| \leqq M \exp [v b(z)], \quad z \in E, \quad M=\text { const, }
$$

$$
\left|Q_{v}(z)\right| \leqq M \psi^{v}(z, E, b), \quad z \in C^{n} .
$$

One obtains easily also the following properties of $\psi$.

$1^{\circ}$ Function $\psi(z, E, b)$ is given by

$$
\psi(z, E, b)=\lim _{v \rightarrow \infty}\left\{\sup _{Q_{v} \in A_{v}}\left|Q_{v}(z)\right|^{1 / v}\right\}, \quad z \in C^{n},
$$


where $A_{v}=A_{v}(E, b)$ denotes the family of all homogeneous polynomials $Q_{v}(z)$ of degree $v$ such that $\left|Q_{v}(z)\right| \leqq \exp [v b(z)]$ for $z \in E$.

$2^{\circ} \psi(z, E, b)$ is an absolutely homogeneous function of order 1 , i.e., $\psi(\lambda z, E, b)$ $=|\lambda| \psi(z, E, b)$ for $z \in C^{n}$ and for any complex $\lambda$.

$3^{\circ}$ There exists a positive number $m$ which depends only on $E$ and $b$ such that

$$
\psi(z, E, b) \geqq m\left(\left|z_{1}\right|+\ldots+\left|z_{n}\right|\right), \quad z \in C^{n} .
$$

$4^{\circ}$ If $\psi(z, E, b)$ is bounded on the unit sphere $\{z \mid\|z\| \leqq 1\}$ and $\psi^{*}(z, E, b)$ $=\lim \sup _{z \prime \rightarrow z} \psi\left(z^{\prime}, E, b\right)$, then $\log \psi^{*}(z, E, b)$ is plurisubharmonic in $C^{n}$.

$5^{\circ}$ If $E_{k}, k=1,2, \ldots, n$, is a bounded closed set of positive logarithmic capacity, $z_{1}^{0} \neq 0$ is a fixed point of the $\left(z_{1}\right)$-plane and $E=\left\{z_{1}^{0}\right\} \times E_{2} \times \ldots \times E_{n}$, then there is a number $M>0$ such that

$$
\psi(z, E, b) \leqq M\left(\left|z_{1}\right|+\ldots+\left|z_{n}\right|\right), \quad z \in C^{n} .
$$

Given an arbitrary compact set $E \subset C^{n}$, let $H(z)=H(z, E)$ be defined by

$$
H(z)=\lim _{v \rightarrow \infty}\left\{\sup \left(\left|a_{\mu_{1} \ldots \mu_{n}} z_{1}^{\mu_{1}} \ldots z_{k}^{\mu}\right|\right)^{1 / v}\right\}, \quad z \in C^{n},
$$

where sup is taken over all the monomials $a_{\mu_{1} \ldots \mu_{n}} z_{1}^{\mu_{1}} \ldots z_{n}^{\mu_{n}}$ of degree $v=\mu_{1}+\ldots$ $+\mu_{n}$ such that

$$
\left|a_{\mu_{1} \ldots \mu_{n}} z_{1}^{\mu_{1}} \ldots z_{n}^{\mu_{n}}\right| \leqq 1 \text { for } z \in E .
$$

THEOREM 2. If $E \subset C^{n}$ is a compact Reinhardt circular set, then

$$
\Phi(z, E, 0)=\max (1, \psi(z, E, 0))=\max (1, H(z)), \quad z \in C^{n} \text {. }
$$

Proof. Let

$$
P_{v}(z)=\sum_{k=0}^{v}\left(\sum_{\mu_{1}+\ldots+\mu_{n}=k} a_{\mu_{1} \ldots \mu_{n}} z_{1}^{\mu_{1} \ldots z_{n}^{\mu_{n}}}\right)
$$

be a polynomial of degree $v$ such that

$$
\left|P_{v}(z)\right| \leqq 1 \quad \text { for } z \in E \text {. }
$$

Then by the Cauchy inequalities we have (due to the definition of a Reinhardt circular set)

$$
\left|a_{\mu_{1} \ldots \mu_{n}} z_{1}^{\mu_{1}} \ldots z_{n}^{\mu_{n}}\right| \leqq 1 \quad \text { for } \quad z \in E .
$$

Therefore, for the points $z$ such that $H(z) \neq 1$ we have

$$
\left|P_{v}(z)\right| \leqq \sum_{k=0}^{v} C_{n+k-1, k} H_{k}(z) \leqq(v+n)^{n} \sum_{k=0}^{v} H^{k}(z)=(v+n)^{n} \frac{H^{v+1}(z)-1}{H(z)-1} \text {. }
$$

Of course, we have also

whence

$$
\left|P_{v}^{\mu}(z)\right| \leqq(\mu v+n)^{n} \frac{H^{\mu v+1}(z)-1}{H(z)-1}, \quad \mu=1,2, \ldots, \quad H(z) \neq 1,
$$

$$
\left|P_{v}(z)\right| \leqq \lim _{\mu \rightarrow \infty}\left[(\mu v+n)^{n} \frac{H^{\mu v+1}(z)-1}{H(z)-1}\right]^{1 / \mu}=\left\{\begin{array}{cl}
H^{v}(z), & \text { if } H(z)>1 \\
1, & \text { if } H(z)<1
\end{array}\right.
$$

Thus, due to Theorem $2, \S 6$, we have 


$$
\Phi(z, E, 0) \leqq \max (1, H(z)), \quad z \in C^{n} .
$$

On the other hand, it follows from Theorem 2, $\S 6$, from $1^{\circ}$ of this section and from the definition of $H(z)$ that

$$
H(z) \leqq \psi(z, E, 0) \leqq \Phi(z, E, 0), \quad z \in C^{n} .
$$

Now (11) follows directly from (12) and (13).

THEOREM 3. If $E \subset C^{n}$ is a compact circular set, then

$$
\Phi(z, E, 0)=\max (1, \psi(z, E, 0)), \quad z \in C^{n} .
$$

Proof. If

$$
P_{v}(z)=\sum_{k=0}^{v} \sum_{\mu_{1}+\ldots+\mu_{n}=k} a_{\mu_{1}} \ldots \mu_{n} z_{1}^{\mu_{1}} \ldots z_{n}^{\mu_{n}}=\sum_{k=0}^{v} Q_{k}(z)
$$

is an arbitrary polynomial of degree $v$ such that

$$
\left|P_{v}(z)\right| \leqq 1 \text { for } z \in E,
$$

then the function $\omega_{v}(\lambda)=\sum_{k=0}^{v} Q_{k}(\lambda \dot{z})=\sum_{k=0}^{v} Q_{k}(\dot{z}) \lambda^{k}$ is a polynomial in $\lambda$ of degree $v$. If $\dot{z} \in E$, then $\left|\omega_{v}(\lambda)\right| \leqq 1$ for $|\lambda|=1$. Therefore, by the Cauchy inequalities

$$
\left|Q_{k}(\dot{z})\right| \leqq 1, \quad \dot{z} \in E, \quad k=0,1, \ldots, v,
$$

whence due to Lemma 1

Therefore,

$$
\left|Q_{k}(z)\right| \leqq \psi^{k}(z, E, 0), \quad z \in C^{n}, \quad k=0,1, \ldots, v .
$$

$$
\left|P_{v}(z)\right| \leqq \frac{\psi^{v+1}(z)-1}{\psi(z)-1} \text {, as } \psi(z, E, 0) \neq 1, \quad v=1,2, \ldots
$$

By a familiar reasoning this inequality, along with the fact that any homogeneous. polynomial of degree $v$ is also an ordinary polynomial of degree $v$, implies (14)

REMARK. One may prove [19] that $H(z)$ is an upper envelope of all absolutely homogenous functions of order 1 which are $\leqq 1$ on $E$ and which are convex with respect to $\xi_{k}=\log \left|z_{k}\right|, k=1,2, \ldots, n$. Moreover, if $E$ is a Reinhardt circular set such that for any $z=\left(z_{1}, \ldots, z_{n}\right) \in E$ we have $z_{i} \neq 0, i=1, \ldots, n$, then $H(z)=H(z, E)$ is continuous in $C^{n}$.

EXAmples. 1. If $E=\{z \mid\|z\|=r\}$, then $H(z)=\psi(z)=\|z\| / r, \Phi(z)$ $=\max (1,\|z\| / r), \quad z \in C^{n}$.

2. If $E=\left\{z|\|| z_{1} /\left.a_{1}\right|^{\alpha}+\ldots+\left|z_{n} / a_{n}\right|^{\alpha}=r\right\}$, then

$$
H(z)=\psi(z)=\left[\frac{1}{r} \sum_{i=1}^{n}\left|\frac{z_{i}}{a_{i}}\right|^{\alpha}\right]^{1 / \alpha}, \quad z \in C^{n} .
$$

3. If $\left.E=\left\{\left(z_{1}, z_{2}\right)|| z_{1}\left|=\xi_{1},\right| z_{2} \mid=\eta_{1}\right\} \cup\left\{\left(z_{1}, z_{2}\right)|| z_{1}\left|=\xi_{2},\right| z_{2} \mid=\eta_{2}\right)\right\}$, $0<\xi_{1}<\xi_{2}, 0<\eta_{2}<\eta_{1}$, then there exist constants $\alpha, \beta$ and $\gamma$ such that 


$$
H(z)=\psi(z)=\max \left(\frac{\left|z_{1}\right|}{\xi_{2}}, \frac{\left|z_{2}\right|}{\eta_{1}}, \frac{\left|z_{1}\right|^{\alpha}\left|z_{2}\right|^{\alpha}}{\gamma}\right), \quad z \in C^{2} .
$$

10. Generalization of the Bernstein-Walsh theorems. In this section we shall always assume that $E$ is a compact subset of $C^{n}$ such that the function $\Phi(z)=\Phi(z, E, 0)$ is continuous in $C^{n}$ and $C E=D_{\infty}$.

Given any $\rho>1$ we define $E_{\rho}$ and and $C_{\rho}$ by

$$
E_{\rho}=\{z \mid \Phi(z)<\rho\}, \quad C_{\rho}=\{z \mid \Phi(z)=\rho\} .
$$

Since by assumption $\Phi(z)$ is continuous, then $E_{\rho}$ is open and $C_{\rho}$ is the boundary of $E_{\rho}$, because $\Phi(z)$ being plurisubharmonic in $C^{n}$ cannot attain its maximum in the interior of a domain without being constant. But $\Phi(z)=\Phi(z, E, 0)>\|z\| / r$ for sufficiently large $r>0$, so $\Phi(z) \not \equiv$ const.

Given the function $f(z)$ defined and bounded on $E$, denote by $R$ the largest real number such that there exists a function $F(z)$ holomorphic in $E_{R}$ and equal to $f(z)$ on $E$.

We say that the sequence of polynomials $\left\{P_{v}(z)\right\}$, where $P_{v}$ is of degree $v$, converges maximally to $f(z)$ on $E$, if

$$
\lim _{v \rightarrow \infty} \sup _{z \in E}\left(\max _{z \in E}\left|f(z)-P_{v}(z)\right|\right)^{1 / v}=\frac{1}{R} .
$$

THEOREM 1. If the polynomials $P_{v}(z)$ of respective degrees $v$ satisfy the condition

$$
\lim \sup _{v \rightarrow \infty}\left|f(z)-P_{v}(z)\right|^{1 / v} \leqq \frac{1}{R}, \quad R>1, \quad z \in E,
$$

and if $R_{1} \in(1, R)$, then the sequence $\left\{P_{v}(z)\right\}$ is uniformly convergent in $E_{R_{1}}$.

Proof. The difference $P_{v+1}-P_{v}$ is a polynomial of degree $v+1$; therefore by Lemma $1, \S 6$,

$$
\left|P_{v+1}(z)-P_{v}(z)\right| \leqq\left[\max _{z \in E}\left|P_{v+1}(z)-P_{v}(z)\right|\right] \Phi^{v+1}(z), \quad z \in C^{n} .
$$

$$
\max _{z \in E}\left|P_{v+1}(z)-P_{v}(z)\right| \leqq \max _{z \in E}\left|P_{v+1}-f\right|+\max _{z \in E}\left|P_{v}-f\right| .
$$

If $\varepsilon>0$ is so small that $\left(R_{1} / R\right)(1+\varepsilon)<1$, then for sufficiently large $N$, we have and further, by (4) and (5),

$$
\max _{\boldsymbol{z} \in \boldsymbol{E}}\left|f-P_{v}\right| \leqq\left(\frac{1+\varepsilon}{R}\right)^{v}, \quad v>N,
$$

whence

$$
\left|P_{v+1}-P_{v}\right| \leqq 2\left(\frac{1+\varepsilon}{R}\right)^{v} \Phi^{v+1}(z), \quad z \in C^{n}, \quad v \geqq N,
$$

$$
\left|P_{v+1}-P_{v}\right| \leqq 2 R_{1}\left[\frac{(1+\varepsilon) R_{1}}{R}\right]^{v}, \quad z \in C_{R_{1}}, \quad v \geqq N .
$$

Therefore, the series $P_{0}+\sum_{k=0}^{\infty}\left(P_{k+1}-P_{k}\right)$ converges uniformly in $\bar{E}_{R_{1}}$. Since $P_{0}+\sum_{k=0}^{v}\left(P_{k+1}-P_{k}\right)=P_{v}$, the proof is completed. 
For polynomials in one variable Theorem 1 is due to Bernstein and Walsh (see [27, p. 78]).

REMARK. After having Lemma $1, \S 6$, the proof of Theorem 1 is the same as the proof of the corresponding theorem for polynomials in one variable. However, the proof of the lemma differs from the well-known proof of the corresponding lemma in the theory of one variable, which is based on the maximum principle for harmonic functions.

THEOREM 2. If $f(z)$ is a holomorphic function on $E$, then there exists a sequence of polynomials $P_{v}(z)$ of respective degrees $v$ which converges maximally to $f(z)$.

Proof. $1^{\circ}$ Suppose $E$ is circular. By Theorem 9.3 and due to $2^{\circ}, \S 9, E_{R}$ is also a circular domain. If the function $f(z)$ is holomorphic in $E_{R}$, then it may be developed in a series of homogeneous polynomials

$$
f(z)=\sum_{\nu=0}^{\infty} Q_{v}(z), \quad z \in E_{R},
$$

$Q_{v}(z)$ being a homogeneous polynomial of degree $v$. If $R_{1} \in(1, R)$, then $E_{R_{1}}=E_{R_{1}}$ $+C_{R_{1}}$ is a compact subset of $E_{R}$ and the series (6) is uniformly absolutely convergent on $\bar{E}_{R_{1}}$. Therefore, there is a constant $M>0$ such that

$$
\left|Q_{v}(z)\right| \leqq M, \quad v=0,1, \ldots, \quad z \in E_{R_{1}} .
$$

In virtue of Lemma $1, \S 9$, we have

$$
\left|Q_{v}(z)\right| \leqq M \psi^{v}\left(z, E_{R_{1}}, 0\right), \quad z \in C^{n}, \quad v=0,1, \ldots,
$$

whence due to the homogeneity of $\psi\left(z, E_{R_{1}}, 0\right)$ and $\psi(z, E, 0)$ we have

$$
\left|Q_{v}(z)\right| \leqq M \frac{1}{R_{1}^{v}} \psi^{v}(z, E, 0), \quad z \in C^{n}
$$

Let $P_{v}(z)=\sum_{k=0}^{v} Q_{k}(z)$. Then

(7) $\left|f(z)-P_{v}(z)\right|=\left|\sum_{k=v+1}^{\infty} Q_{k}(z)\right| \leqq M \sum_{k=v+1}^{\infty} \frac{1}{R_{1}^{k}}=\frac{M}{R_{1}^{v+1}\left(1-1 / R_{1}\right)}, \quad z \in E$,

whence due to the arbitrariness of $R_{1} \in(1, R)$, we have

$$
\lim _{v \rightarrow \infty} \sup _{z \in E}\left(\max _{z \in E}\left|f-P_{v}\right|\right)^{1 / v} \leqq \frac{1}{R}
$$

and the inequality lim $\sup _{v \rightarrow \infty}\left(\max _{z \in E}\left|f-P_{v}\right|\right)^{1 / v}<1 / R$ is impossible, as follows immediately from Theorem 1, Q.E.D.

$2^{\circ}$ Suppose $E$ is arbitrary. Since by our general assumption the function $\Phi(z)=\Phi(z, E, 0)$ is continuous and, on the other hand, $\Phi(z)$ is an upper bound of continuous functions $\Phi_{v}(z)=\left(\max _{(i)}\left|L^{(i)}\left(z, \gamma^{(v)}\right)\right|\right)^{1 / v}$, therefore by Dini's theorem the sequence $\Phi_{v}$ converges uniformly to $\Phi(z)$ on any closed bounded subset of $C^{n}$. 
Let $R_{1}>1$ and $\varepsilon>0$ be arbitrary real numbers such that $R_{1}+\varepsilon<R-\varepsilon$. Since $\Phi_{v}(z)$ converges to $\Phi(z)$ uniformly on $E_{R}$, there exists integer $m$ such that

$$
\Phi_{m}(z)<R_{1}+\varepsilon \quad \text { for } z \in C_{R_{1}}
$$

and

$$
\Phi_{m}(z)>R-\varepsilon \quad \text { for } z \in C_{R} \text {. }
$$

Let

$$
G=\left\{z|| L^{(i)}\left(z, \gamma^{(m)}\right) \mid<(R-\varepsilon)^{m}, \quad i=1,2, \ldots, m_{*}\right\}, m_{*}=C_{m+n, n}
$$

Of course,

$$
G=\left\{z \mid \Phi_{m}(z)<R-\varepsilon\right\} .
$$

In virtue of (8) and (9) and since $R_{1}+\varepsilon<R-\varepsilon$, we have

$$
C_{R_{1}} \subset G \text { and } G \subset E_{R} \text {. }
$$

Let $m^{\prime}$ denote the smallest integer such that $G^{\prime}$ defined by

$$
G^{\prime}=\left\{z|| L^{(i k)}\left(z, \gamma^{(m)}\right) \mid<(R-\varepsilon)^{m}, \quad k=1,2, \ldots, m^{\prime}\right\}
$$

is identical with $G$. Without any loss of generality we may assume that $i_{k}=k$ for $k=1,2, \ldots, m^{\prime}$. Thus

$$
G=\left\{z|| L^{(i)}\left(z, \gamma^{(m)}\right) \mid<(R-\varepsilon)^{m}, \quad i=1,2, \ldots, m^{\prime}\right\} .
$$

By assumption (by definition of $R$ ) the function $f(z)$ is holomorphic in $E_{R}$. Then it is holomorphic by (10) in $G$. In the following we shall write $L^{(i)}(z)$ instead of $L^{(i)}\left(z, \gamma^{(m)}\right)$. By a theorem of A. Weil $[25 ; 26]$,

or

$$
f(z)=\frac{1}{(2 \pi i)^{n}} \sum_{1 \leqq i_{1}<i_{2}<\ldots<i_{n} \leqq m^{\prime}} \int_{\sigma_{i_{1} \ldots i_{n}}} \frac{\delta_{i_{1} \ldots i_{n}}(z, \zeta) f(\zeta) d \zeta_{1} \ldots d \zeta_{n}}{\prod_{k=1}^{m^{\prime}}\left[L^{\left(i_{k}\right)}(z)-L^{\left(i_{k}\right)}(\zeta)\right]}, z \in G,
$$

(12) $f(z)=\sum_{1 \leqq i_{1}<\ldots<i_{n} \leqq m^{\prime}} \sum_{k_{1}, \ldots, k_{n}=0}^{\infty} P_{i_{1} \ldots i_{n} k_{1} \ldots k_{n}}(z)\left[L^{\left(i_{1}\right)}(z)\right]^{k_{1}} \ldots\left[L^{\left(i_{n}\right)}(z)\right]^{k_{n}}, \quad z \in G$, where

$$
P_{i_{1} \ldots i_{n} k_{1} \ldots k_{n}}(z)=\frac{1}{(2 \pi i)^{n}} \int_{\sigma i_{1} \ldots i_{n}} \frac{\delta_{i_{n} \ldots i_{n}}(z, \zeta) f(\zeta) d \zeta_{1} \ldots d \zeta_{n}}{\left.\left[L_{n}\right)(\zeta)\right]^{k_{n}+1} \ldots\left[L^{\left(i_{n}\right)}(\zeta)\right]^{k_{n}+1}}
$$

and $\sigma_{i_{1} \ldots i_{n}}$ denotes some $n$-dimensional part of the boundary of $G$ and $\delta_{i_{1} \ldots i_{n}}(z, \zeta)$ is the determinant

while $P_{i_{k l} l}$ is defined by the relations

$$
\delta_{i_{1} \ldots i_{n}}=\operatorname{det}\left[P_{i_{k} l}\right]_{k, l=1,2, \ldots n} .
$$

$$
L^{(i)}(z)-L^{(i)}(\zeta) \equiv \quad \sum_{l=1}^{n}\left(z_{l}-\zeta_{l}\right) P_{i l}, \quad i=1,2, \ldots, m^{\prime} .
$$

Thus, $P_{i l}(z, \zeta)$ are polynomials of order $m$, both in $z=\left(z_{1}, \ldots, z_{n}\right)$ and in $\zeta=\left(\zeta_{1}, \ldots \zeta_{n}\right)$. The polynomial

$$
Q_{k_{1} \ldots k_{n}}(z)=\sum_{1 \leqq i_{1} \leqq \ldots \leqq i_{n} \leqq m^{\prime}} P_{i_{1} \ldots i_{n} k_{1} \ldots k}(z)\left[L^{\left(i_{1}\right)}(z)\right]^{k_{1}} \ldots\left[L^{\left(i_{n}\right)}(z)\right]^{k_{n}}
$$


is of order $m+m\left(k_{1}+\ldots+k_{n}\right)$. For $z \in E$ we have $\left|L^{(i)}(z)\right|=\left|L^{(i)}\left(z, \gamma^{(m)}\right)\right| \leqq 1$, $i=1,2 \ldots, m_{*}$. Therefore, due to (10), (11) and (13), we have

$$
\left|Q_{k_{1} \ldots k_{n}}(z)\right| \leqq M /(R-\varepsilon)^{m\left(k_{1}+\ldots+k_{n}+n\right)}, \quad z \in E,
$$

$M$ being a constant which depends only on $R_{1}$ and $f$. Let

By (12) and (14) we have

$$
P_{m(v+n)}(z)=\sum_{k_{1}+\ldots+k_{n} \leqq v} Q_{k_{1} \ldots k_{n}}(z), \quad v=1,2, \ldots .
$$

$$
\begin{aligned}
\left|f(z)-P_{m(v+n)}(z)\right| & \leqq M \sum_{k_{1}+\ldots+k_{n} \geqq v+1} 1 /(R-\varepsilon)^{m\left(k_{1}+\ldots+k_{n}+n\right)} \\
& \leqq M_{1} /(R-\varepsilon)^{m(v+1+n)}, \quad z \in E,
\end{aligned}
$$

$M_{1}$ being a constant. Therefore,

$$
\limsup _{v \rightarrow \infty}\left\{\max _{z \in E}\left|f-P_{m(v+n)}\right|\right\}^{1 / m(v+n)} \leqq \frac{1}{R-\varepsilon} .
$$

The polynomials $\widetilde{P}_{\mu}$, where $\tilde{P}_{\mu}=P_{m(v+n)}$ for $m(v+n) \leqq \mu<m(v+n), v=1,2, \ldots$, and $\tilde{P}_{\mu} \equiv 0$ for $\mu=1,2, \ldots, m n$, are of respective degrees $\mu$ and moreover

$$
\lim _{v \rightarrow \infty} \sup _{z \in E}\left(\max _{z \in}\left|f-\tilde{P}_{v}\right|\right)^{1 / v} \leqq \frac{1}{R-\varepsilon} .
$$

The sequence $\left\{\widetilde{P}_{v}\right\}$ depends on $\varepsilon$ and $R_{1}$. Letting now $R_{1}$ go to $R$ and $\varepsilon$ to 0 we may find by a diagonal process polynomials $P_{v}(z)$ of respective degrees $v$ such that

$$
\lim _{v \rightarrow \infty} \sup _{z \in E}\left(\max _{z \in E}\left|f-P_{v}\right|\right)^{1 / v} \leqq \frac{1}{R} .
$$

Since by Theorem 1 the inequality $\lim \sup _{v \rightarrow \infty}(\ldots)^{1 / v}<1 / R$ cannot hold, the proof of the theorem is completed.

In the case of one variable Theorem 2 is due to Faber, Bernstein, Szegö and Walsh (for reference see [27]).

Let $\pi_{v}(z)$ denote the Tchebycheff polynomial of degree $v$ of the best approximation to $f(z)$ on $E$. An immediate consequence of Theorems 1 and 2 is

THEOREM 3. A necessary and sufficient condition that the function $f(z)$ be holomorphic in $E_{\rho}, \rho>1$, is that

$$
\limsup _{v \rightarrow \infty}\left(\max _{z \in E}\left|f(z)-\pi_{v}(z)\right|\right)^{1 / v} \leqq \frac{1}{\rho} .
$$

For polynomials in one variable this theorem is well known [27]. In the case that $E$ is a Cartesian product of linear intervals $E_{k}=\left\{z_{k} \mid-1 \leqq z_{k}=x_{k} \leqq 1\right\}$, $k=1,2, \ldots, n$, Theorem 3 is due to Sapogov [17].

\section{Interpolation at extremal points.}

THEOREM 1. If the complement of $E$ is connected, $\Phi(z)=\Phi(z, E, 0)$ is continuous in $C^{n}$ and the function $f(z)$ is holomorphic on $E$, then the sequence of interpolating polynomials 


$$
L_{v}(z, f)=\sum_{i=1}^{v_{*}} f\left(\gamma_{i}^{(v)}\right) L^{(i)}\left(z, \gamma^{(v)}\right), \quad v=1,2 \ldots,
$$

where $\gamma^{(v)}=\left\{\gamma_{1}^{(v)}, \ldots, \gamma_{v_{*}}^{v)}\right\}$ is the vth extremal system of $E$ with respect to $b(z) \equiv 0$ converges maximally to $f(z)$.

Proof. By Theorem 10.2 there is a sequence of polynomials $P_{v}(z)$ of respective degrees $v$ which converges maximally to $f(z)$, i.e.,

$$
\limsup _{v \rightarrow \infty}\left(\max _{z \in E}\left|f-P_{v}\right|\right)^{1 / v}=\frac{1}{R},
$$

where $R>1$ is the largest number such that $f(z)$ is holomorphic in $E_{R}$. If $R_{1} \subset(1, R)$, there is a const $M>0$ such that

$$
\left|f(z)-P_{v}(z)\right| \leqq \frac{M}{R_{1}^{v}}, \quad v=1,2, \ldots, \quad z \in E .
$$

Since

$$
L_{v}(z, f)-P_{v}(z) \equiv \sum_{i=1}^{v_{*}}\left[f\left(\gamma_{i}^{(v)}\right)-P_{v}\left(\gamma_{i}^{(v)}\right)\right] L^{(i)}\left(z, \gamma^{(v)}\right), \quad v=1,2, \ldots,
$$

then by (3) and (6.7)

$$
\left|L_{v}(z, f)-P_{v}(z)\right| \leqq \frac{\alpha_{v} v_{*} M}{R_{1}^{v}}, \quad v=1,2, \ldots, \quad z \in E .
$$

It follows from (3) and (4) that

whence by (5.2) we have

$$
\left|f(z)-L_{v}(z, f)\right| \leqq \frac{M}{R_{1}^{v}}\left(1+v_{*} \alpha_{v}\right), \quad v=1,2, \ldots, \quad z \in E,
$$

$$
\lim _{v \rightarrow \infty} \sup \left(\max _{z \in E}\left|f(z)-L_{v}(z, f)\right|\right)^{1 / v} \leqq \frac{1}{R_{1}} .
$$

Due to the arbitrariness of $R_{1}$ and because of Theorem 10.1 the last inequality implies our theorem, Q.E.D.

If $E \subset C^{1}$, Theorem 1 is due to Fekete [5] (see also [27, p. 171]).

Let now $E=E_{1} \times \ldots \times E_{n}$, where $E_{k}$ is regular and has a connected complement. Let

$$
\omega_{k v}\left(z_{k}\right)=\left(z_{k}-x_{k 0}\right) \ldots\left(z_{k}-x_{k v-1}\right), \quad v=1,2, \ldots, \quad k=1,2, \ldots, n,
$$

where

$$
x_{k 0}, x_{k 1}, \ldots, x_{k v}, \ldots,
$$

denotes a sequence of Leja's extremal points of $E_{k}, k=1,2, \ldots, n$, respectively (see Lemma 8.1). Suppose $f(z)$ is holomorphic in the Cartesian product $E_{R_{1} \ldots R_{n}}$ of the domains

$$
E_{R_{k}}=\left\{z_{k} \mid \Phi\left(z_{k}, E_{k}, 0\right)<R_{k}\right\}, \quad R_{k}>1, \quad k=1,2, \ldots, n .
$$

Let

$$
C_{k}=\left\{z_{k} \mid \Phi\left(z_{k}, E_{k}, 0\right)=R_{k}^{\prime}\right\}, \quad \text { where } R_{k}^{\prime} \in\left(1, R_{k}\right), \quad k=1,2, \ldots, n
$$


THEOREM 2. The Newton's series of $f(z)$

$$
f(z) \sim a_{0}+\sum_{l=1}^{\infty} \sum_{i_{1}+\ldots+i_{n}=l} a_{i_{1} \ldots i_{n}} \prod_{k=1}^{n} \omega_{k i_{k}}\left(z_{k}\right),
$$

converges uniformly on any closed subset of $E_{R_{1} \ldots R_{n}}$.

Proof. Since by (3.10) we have

$$
a_{i_{1} \ldots i_{n}}=\frac{1}{(2 \pi i)^{n}} \int_{c_{1}} \ldots \int_{c_{n}} \frac{f\left(\zeta_{1}, \ldots, \zeta_{n}\right) d \zeta_{1} \ldots d \zeta_{n}}{\prod_{k=1}^{n}\left(\zeta_{k}-x_{k i_{k}}\right) \prod_{k=1}^{n} \omega_{k i_{k}}\left(\zeta_{k}\right)},
$$

and $C_{k} \cap E=\emptyset, k=1,2, \ldots, n$, then

$$
\left|a_{i_{1} \ldots i_{n}}\right| \leqq M / \prod_{k=1}^{n} \min _{\zeta k \in C_{k}}\left|\omega_{k i_{k}}\left(\zeta_{k}\right)\right|, \quad i_{1}, i_{2}, \ldots, i_{n}=0,1, \ldots,
$$

$M$ depending only on $C_{1} \times C_{2} \times \ldots \times C_{n}$ and on $f$. We know (see Lemma 8.1) that the sequence $\left\{\left(\left|\omega_{k v}\left(z_{k}\right) / \omega_{k v}\left(x_{k v}\right)\right|\right)^{1 / \nu}\right\}$ converges uniformly to $\Phi\left(z_{k}, E_{k}, 0\right)$ on any compact subset of the $\left(z_{k}\right)$-plane. Therefore, given $\varepsilon_{k}>0$ sufficiently small, there is a constant $M_{1}>0$ such that

$$
\left|a_{i_{1} \ldots i_{n}}\right| \leqq M_{1} /\left[\prod_{k=1}^{n}\left(R_{k}^{\prime}-\delta_{k}\right)^{i_{k}}\left|\omega_{k i_{k}}\left(x_{k i_{k}}\right)\right|\right],
$$

whence it follows that series (9) is majorized by the series

$$
\left|a_{0}\right|+M_{1} \sum_{l=1}^{\infty} \sum_{i_{1}+\ldots+i_{n}=l} \prod_{k=1}^{n} \frac{\left|\omega_{k i_{k}}\left(z_{k}\right)\right|}{\left.\left|\omega_{k i_{k}}\left(x_{k i_{k}}\right)\right| R_{k}^{\prime}-\varepsilon_{k}\right) i_{k}} .
$$

Let $\varepsilon_{k}=\varepsilon_{1}\left(R_{1}^{\prime} / R_{k}^{\prime}\right), k=2, \ldots, n$, then

$$
\frac{R_{k}^{\prime}-2 \varepsilon_{k}}{R_{k}^{\prime}-\varepsilon_{k}}=\frac{R_{1}^{\prime}-2 \varepsilon_{1}}{R_{1}^{\prime}-\varepsilon_{1}}
$$

There is a constant $M_{2}>0$ such that

$$
\left|\frac{\omega_{k i_{k}}\left(z_{k}\right)}{\omega_{k i_{k}}\left(x_{k i_{k}}\right)}\right| \leqq M_{2}\left(R_{k}^{\prime}-2 \varepsilon_{k}\right)^{i_{k}} \text { for } z_{k} \in\left\{z_{k} \mid \Phi\left(z_{k}, E_{k}, 0\right)=R_{k}^{\prime}-3 \varepsilon_{k}\right\}
$$

Then the series

$$
\left|a_{0}\right|+M_{1} M_{2} \sum_{l=1}^{\infty} \sum_{i_{1}+\ldots+i_{n}=l}\left(\frac{R_{1}^{\prime}-2 \varepsilon_{1}}{R_{1}^{\prime}-\varepsilon_{1}}\right)^{i_{1}+\ldots+i_{n}}
$$

is convergent and it majorizes the series (10) (and therefore the series (9) for $z$ in the Cartesian product of the sets $\left\{z_{k} \mid \Phi\left(z_{k}, E_{k}, 0\right)=R_{k}^{\prime}-3 \varepsilon_{k}\right\}$. Due to the arbitrariness of $\varepsilon_{k}$ and of $R_{k}^{\prime}, k=1, \ldots, n$, this implies that the series (9) is convergent uniformly on any closed subset of $E_{R_{1} \ldots R_{n}}$ to some holomorphic function $g(z)$. But due to Lemma 3.2, we have

$$
g\left(x_{1 i_{1}}, \ldots, x_{n i_{n}}\right)=f\left(x_{1 i_{1}}, \ldots, x_{n i_{n}}\right), \quad i_{1}, i_{2}, \ldots, i_{n}=0,1,2, \ldots,
$$

whence $g(z) \equiv f(z)$. The proof is completed. 
12. Existence of the limit $u(z, E, b)=\lim _{\lambda \downarrow 0}(1 / \lambda) \log \Phi(z, E, \lambda b) / \Phi(z, E, 0)$. Let $E$ be a compact set in $C^{n}$ such that $\Phi(z, E, 0)$ is finite at any point of $C^{n}$. If $b(z)$ is an arbitrary real function defined and bounded on $E$ and if $\lambda$ is a real number $>0$, then by (7.1)

$$
\inf _{\zeta \in E} b(\zeta)=b_{0} \leqq \frac{1}{\lambda} \log \frac{\Phi(z, E, \lambda b)}{\Phi(z, E, 0)} \leqq B_{0}=\sup _{\zeta \in E} b(\zeta), \quad z \in C^{n} .
$$

We shall prove the following inequality

$$
\left[\frac{\Phi(z, E, \lambda b)}{\Phi(z, E, 0)}\right]^{1 / \lambda} \leqq\left[\frac{\Phi\left(z, E, \lambda^{\prime} b\right)}{\Phi(z, E, 0)}\right]^{1 / \lambda^{\prime}}, \quad 0<\lambda^{\prime} \leqq \lambda, z \in C^{n} .
$$

At first let $\lambda$ and $\lambda^{\prime}$ be rational

We have

$$
\lambda^{\prime}=\frac{p^{\prime}}{q^{\prime}}<\frac{p}{q}=\lambda
$$

Therefore, by (7.8)

$$
\frac{p^{\prime}}{q^{\prime}} b=\frac{p^{\prime} q}{q^{\prime} p} \cdot \frac{p}{q} b=\frac{1}{q^{\prime} p}\left[p^{\prime} q \frac{p}{q} b+\left(q^{\prime} p-p^{\prime} q\right) 0\right] .
$$

$$
\Phi^{p^{\prime} q}\left(z, E, \frac{p}{q} b\right) \Phi^{q^{\prime} p-p^{\prime} q}(z, E, 0) \leqq \Phi^{q^{\prime} p}\left(z, E, \frac{p^{\prime}}{q^{\prime}} b\right),
$$

whence we obtain (1) for $\lambda=p / q$ and $\lambda^{\prime}=p^{\prime} / q^{\prime}$.

To prove (1) for arbitrary $\lambda$ and $\lambda^{\prime}$ let $\lambda_{v}$ and $\lambda_{v}^{\prime}$ be rational numbers such that

$$
\lambda_{v}^{\prime} \leqq \lambda^{\prime} \leqq \lambda \leqq \lambda_{v}, \quad \lambda_{v}^{\prime} \rightarrow \lambda^{\prime}, \quad \lambda_{v} \rightarrow \lambda,
$$

and let $b_{0}=\inf _{z \in E} b(z)$. We have $b(z)+b_{0} \geqq 0$. Due to (7.7)

and

$$
\left[\frac{\Phi\left(z, E, \lambda\left(b+b_{0}\right)\right)}{\Phi(z, E, 0)}\right]^{1 / \lambda_{v}} \leqq\left[\frac{\Phi\left(z, E, \lambda_{v}\left(b+b_{0}\right)\right)}{\Phi(z, E, 0)}\right]^{1 / \lambda_{v}}
$$

$$
\left[\frac{\Phi\left(z, E, \lambda_{v}^{\prime}\left(b+b_{0}\right)\right)}{\Phi(z, E, 0)}\right]^{1 / \lambda^{\prime}} \leqq\left[\frac{\Phi\left(z, E, \lambda^{\prime}\left(b+b_{0}\right)\right)^{1 / \lambda^{\prime}{ }_{v}}}{\Phi(z, E, 0)}\right] .
$$

Since $\lambda_{v}$ and $\lambda_{v}^{\prime}$ are rational, we have by (7.5)

$$
\exp \left(\frac{\lambda}{\lambda_{v}} b_{0}\right)\left[\frac{\Phi(z, E, \lambda b)}{\Phi(z, E, 0)}\right]^{1 / \lambda_{v}} \leqq \exp \left(\frac{\lambda^{\prime}}{\lambda_{v}^{\prime}} b_{0}\right)\left[\frac{\Phi\left(z, E, \lambda^{\prime} b\right)}{\Phi(z, E, 0)}\right]^{1 / \lambda^{\prime}},
$$

whence the inequality (1) follows in an obvious way.

THEOREM 1. If $E \subset C^{n}$ is a compact set, and if $b(z)$ is a real function defin$e d$ and bounded on $E$, then there exists a finite limit

$$
u(z, E, b)=\lim _{\lambda \downarrow 0} \frac{1}{\lambda} \log \frac{\Phi(z, E, \lambda b)}{\Phi(z, E, 0)}
$$

at any point $z \in C^{n}$ such that $\Phi(z, E, 0)$ is finite. Moreover, the function 


$$
u^{*}(z)=\lim _{z^{\prime} \rightarrow z} \sup u\left(z^{\prime}, E, b\right)
$$

is plurisubharmonic at any interior point of

$$
\mathscr{E}_{1}=\{z \mid \Phi(z, E, 0)=1\} .
$$

Proof. The existence of the limit (2) follows directly from (1). The function $u^{*}(z)$ is, for $z \in \mathscr{E}_{1}$, an upper envelope of plurisubharmonic functions

$$
\frac{1}{\lambda} \log \Phi^{*}(z, E, \lambda b)=\limsup _{z^{\prime} \rightarrow z} \frac{1}{\lambda} \log \Phi\left(z^{\prime}, E, \lambda b\right) ;
$$

therefore it is plurisubharmonic at any interior point if $\mathscr{E}_{1}$, Q.E.D.

If $E$ is a line segment in $C^{1}$ and if $E$ is a Jordan curve in $C^{1}$, the existence of the limit (2) for $z \in E$ has been proved in [11] and [8], respectively. The method of proof used by the authors of these papers was based on the generalized approximation theorem of Weierstrass.

Inoue in [8] and Leja in [13] have proved that if $E$ is a Jordan curve in $C^{1}$ and $b(z)$ is continuous, then $u(z, E, b)$ is a solution of the Dirichlet boundary value problem for the interior of $E$ with boundary values $b(z)$. The author of this paper has shown in his thesis [21] that if $E$ is a boundary of a domain $D$ which contains the point $\infty$ in its interior and if $b(z)$ is real and bounded, then $u(z, E, b)$ is Perron's generalized solution of the Dirichlet problem for any component of $C E$ with boundary values $b(z)$.

In the next section we shall establish the relationship of $u(z, E, b)$ to Bremerman's [4] solution of the Dirichlet problem for plurisubharmonic functions in $C^{n}$.

13. The generalized Dirichlet problem for plurisubharmonic functions. Let $D$ be a bounded domain in $C^{n}$ and let $F=F(D)$ denote the Silov boundary of $D$ with respect to plurisubharmonic functions in $\bar{D}$ (plurisubharmonic in a neighborhood of $D$ which may depend on the particular functions). The family of functions plurisubharmonic in a given domain $D$ does not form an algebra. Therefore, there is a natural question of the existence of the Silov boundary with respect to such a family. However, it has been shown in [20] that the Silov boundary exists for separating function families which are closed only with respect to addition (or multiplication). Therefore, the existence of the Silov boundary with respect to plurisubharmonic functions is guaranteed.

Let $E$ be a subset of $\bar{D}$ such that $F \subset E$ and let $b(z)$ be a real bounded function (continuous or not) defined on $E$. Denoted by $A=A(D, E, b)$ the family of all functions $U(z)$ plurisubharmonic in $\bar{D}$ such that

$$
U(z) \leqq b(z) \text { for } z \in E,
$$

we define the upper envelope $V^{*}(z)$ of functions $U$ by

$$
V(z)=\sup _{U \in A} U(z), \quad V^{*}(z)=\lim _{z^{\prime} \rightarrow z} \sup V\left(z^{\prime}\right), \quad z, z^{\prime} \in \bar{D} .
$$


The function $V^{*}(z)$ has been introduced and investigated in [4] for the case that $D$ is a pseudoconvex domain with "smooth" boundary and $b(z)$ is defined and continuous on $E=F$. In $\S 8$ of [4] the connection of $V^{*}(z)$ with the envelope of holomorphy of Hartogs domains has been considered. We want to add what follows to these considera tions. Let

$$
H=\left\{(z, w)|z \in E,| w \mid \leqq e^{-b(z)}\right\}
$$

and

$$
G=\left\{(z, w)|z \in D,| w \mid<e^{-V^{*}(z)}\right\}
$$

We shall prove

LEMMA 1. If the functions $a_{v}(z), v=0,1,2, \ldots$, are holomorphic in a neighborhood of $\bar{D}$ and the series

$$
f(z, w)=\sum_{v=0}^{\infty} a_{v}(z) w^{v}
$$

is uniformly convergent $\left({ }^{3}\right)$ on $H$, then it is uniformly absolutely convergent in any closed subset of the domain $G$.

Proof. There is a constant $M>0$ such that

$$
\left|a_{v}(z) w^{v}\right| \leqq M \quad \text { for } \quad(z, w) \in H, \quad v=0,1, \ldots,
$$

i.e.,

$$
\left|a_{v}(z)\right| \leqq M e^{v b(z)} \quad \text { for } \quad z \in E, \quad v=0,1, \ldots
$$

Since $(1 / v) \log \left|a_{v}(z)\right|$ is plurisubharmonic in $\bar{D}$, therefore by definition of $V^{*}(z)$ we have

$$
\left|\frac{a_{v}(z)}{M}\right|^{1 / v} \leqq e^{V^{*}(z)}, \quad z \in \bar{D}, \quad v=1,2, \ldots,
$$

whence it follows that the series (5) is uniformly absolutely convergent in any compact subset of $G$.

LEMMA 2. There exists a sequence $\left\{V_{k}(z)\right\}$ of plurisubharmonic functions in $\bar{D}$ such that

$$
V_{k}(z)<V(z) \text { for } z \in \bar{D}, \quad k=1,2 \ldots,
$$

and

$$
V^{*}(z)=\left\{\sup _{k=1,2, \ldots} V_{k}(z)\right\}^{*}
$$

Proof. Let $\left\{z_{v}\right\}$ be an arbitrary sequence of points everywhere dense in $D$. Denote by $z_{v k}$ the point of $D$ such that

$$
V^{*}\left(z_{v k}\right)=\max _{\left\|z-z_{v}\right\| \leqq 1 / k} V^{*}(z) .
$$

(3) It is sufficient to assume that $\left|a_{v}(z) w^{v}\right|, v=1,2, \ldots$, are uniformly bounded on $H$. 
For any point $z_{v k}$ one may find a sequence $\left\{z_{v k l}\right\}_{l=1,2 \ldots}$ of points of $D$ such that

$$
\lim _{l \rightarrow \infty} z_{v k l}=z_{v k} \text { and } \lim _{l \rightarrow \infty} V\left(z_{v k l}\right)=V^{*}\left(z_{v k}\right) \text {. }
$$

One can easily check that the set $D^{*}$ of all the points $z_{v k l}, v, k, l=1,2, \ldots$, satisfies the following property

$$
\lim _{z^{\prime} \rightarrow z, z^{\prime} \in D^{*}} \sup V\left(z^{\prime}\right)=V^{*}(z), \quad z \in D .
$$

Let us arrange the points of $D^{*}$ into a sequence, say $\left\{p_{v}^{\prime}\right\}$ and take the sequence $\left\{p_{v}\right\}$ of points

$$
p_{1}^{\prime}, p_{1}^{\prime}, p_{2}^{\prime}, p_{1}^{\prime}, p_{2}^{\prime}, p_{3}^{\prime}, \ldots
$$

By definition of $V(z)$ for any $p_{k}$ there exists a function $V_{k}(z)$ plurisubharmonic in $\bar{D}$ such that

$$
V_{k}(z)<V(z), \quad z \in \bar{D} \text { and } \quad V_{k}(z)>V\left(p_{k}\right)-(1 / k), \quad k=1,2, \ldots
$$

Since any point of $D^{*}$ is repeated infinitely many times in the sequence $\left\{p_{v}\right\}$, then

$$
V_{k}(z)<V(z), \quad z \in \bar{D} \text { and } V(z)=\sup _{k} V_{k}(z), \quad z \in D^{*} .
$$

Therefore, due to (9), the sequence $\left\{V_{k}(z)\right\}$ has all the required properties.

LEMMA 3. Suppose that for the domain $D$ there exists a sequence of domains of holomorphy $\left\{D_{v}\right\}$ such that

$$
D_{v} \supset D_{v+1} \supset \bar{D}, \quad v=1,2, \ldots,
$$

and for any $\varepsilon>0$ there is $v_{0}$ such that

$$
D_{v} \subset D_{\varepsilon}=\left\{z \mid \min _{\zeta_{\varepsilon} \bar{D}}\|z-\zeta\|<\varepsilon\right\} \quad \text { for } v \geqq v_{0} .
$$

Then the function $V^{*}(z)$ is an upper envelope of all the functions $(1 / k) \log |g(z)|$, where $k$ is an integer and $g(z)$ is a function holomorphic in $\bar{D}$ such that

$$
(1 / k) \log |g(z)| \leqq b(z), \text { for } z \in E .
$$

Proof. Without any loss of generality we may assume that the functions $V_{k}(z)$ defined in the proof of Lemma 2 are plurisubharmonic in $D_{k}, k=1,2, \ldots$, respectively. It is known [4] that

$$
H_{k}=\left\{(z, w)\left|z \in D_{k}, \quad\right| w \mid<\exp \left[-V_{k}(z)\right]\right\}, \quad k=1,2, \ldots,
$$

is a domain of holomorphy and there exists the function $f_{k}(z, w)$ holomorphic in $H_{k}$ such that

$$
f_{k}(z, w)=\sum_{v=0}^{\infty} a_{v}^{(k)}(z) w^{v}, \quad(z, w) \in H_{k},
$$


where the $a_{v}^{(k)}(z)$ are holomorphic in $D_{k}$ and the series is uniformly convergent in any compact subset of $H_{k}$. Moreover,

$$
V_{k}(z)=\left\{\limsup _{v \rightarrow \infty} \frac{1}{v} \log \left|a_{v}^{(k)}(z)\right|\right\}^{*}, \quad z \in D_{k} .
$$

By the uniform convergency of (14) there is a constant $M_{k}>0$ which does not depend on $v$ such that

$$
\left|a_{v}^{(k)}(z)\right| \leqq M_{k} \exp \left[v\left(V_{k}(z)+\frac{1}{2 k}\right)\right], \quad z \in \bar{D}, \quad v, k=1,2, \ldots .
$$

It follows from (15) that

$$
\left\{\sup _{v} \frac{1}{v} \log \left|\frac{a_{v}^{(k)}(z)}{M_{k} e^{1 / 2 k}}\right|\right\}^{*}=V_{k}(z) .
$$

Denoting $\left.g_{k v}(z)=a_{v}^{(k)}(z) / M_{k} e^{1 / 2 k}\right)$ we have

and

$$
\frac{1}{v} \log \left|g_{k v}(z)\right| \leqq V_{k}(z), \quad z \in D, \quad k, v=1,2, \ldots,
$$

$$
\left\{\sup _{k}\left\{\sup _{v} \frac{1}{v} \log \left|g_{k v}(z)\right|\right\}^{*}\right\}^{*}=V^{*}(z)
$$

To complete the proof it is enough to show that

$$
V^{*}(z)=\left\{\sup _{k, v} \frac{1}{v} \log \left|g_{k v}(z)\right|\right\}^{*} .
$$

Let $A(z)=\sup _{k, v}(1 / v) \log \left|g_{k v}(z)\right|$. By (12) and (16)

$$
A^{*}(z) \leqq V^{*}(z) \text {. }
$$

The function $A^{*}(z)$ is upper semicontinuous. Therefore, given $z_{0} \in \bar{D}$ and $\varepsilon>0$ there is a number $\delta>0$ such that

$$
A^{*}(z) \leqq A^{*}\left(z_{0}\right)+\varepsilon, \text { for }\left\|z-z_{0}\right\| \leqq \delta, \quad z \in \bar{D} .
$$

Thus

$$
\frac{1}{v} \log \left|g_{k v}(z)\right| \leqq A^{*}(z) \leqq A^{*}\left(z_{0}\right)+\varepsilon, \quad\left\|z-z_{0}\right\|<\delta, \quad z \in \bar{D}, \quad k, v=1,2, \ldots,
$$

whence

$V^{*}(z)=\left\{\sup _{k}\left\{\sup _{v} \frac{1}{v} \log \left|g_{k v}(z)\right|\right\}^{*}\right\}^{*} \leqq A^{*}\left(z_{0}\right)+\varepsilon, \quad\left\|z-z_{0}\right\|<\delta, \quad z \in \bar{D}$.

Since $\varepsilon>0$ is arbitrarily small, we have $V^{*}\left(z_{0}\right) \leqq A^{*}\left(z_{0}\right)$. Therefore, $A^{*}(z) \equiv V^{*}(z)$, Q.E.D.

Putting $E=\bar{D}$, it follows from Lemma 3

COROLlaRY 1. If D satisfies the assumptions of Lemma 3, then the Šlov boundary of $D$ with respect to functions which are plurisubharmonic in $\bar{D}$ is equal to the Silov boundary of $D$ with respect to functions which are holomorphic in $\bar{D}$ (compare $[4, \mathrm{p} .262])$. 
We shall prove also

Corollary 2 (Lemma of Bremermann) [4, p. 256]). If $U(z)$ is plurisubharmonic and continuous in a domain of holomorphy $G$, then for any $G \subset \subset \subset$ and for any $\varepsilon>0$ there exist $k$ functions $f_{1}, \ldots, f_{k}$ holomorphic in $G$ and $k$ positive integers $c_{1}, \ldots, c_{k}$ such that

$$
U(z)-\varepsilon \leqq \sup \left\{\frac{1}{c_{1}} \log \left|f_{1}(z)\right|, \ldots, \frac{1}{c_{k}} \log \left|f_{k}(z)\right|\right\} \leqq U(z), \quad z \in G .
$$

Indeed, there is a domain $D$ which satisfies the conditions of Lemma 3 such that

$$
\tilde{G} \subset D \subset \subset G \text {. }
$$

Therefore, due to the continuity of $U(z)$ there is a finite system of functions $f_{i}(z), i=1,2, \ldots, k$, which satisfy (19) for $z \in D$. Thus Corollary 2 is true.

THEOREM 1. If D satisfies the assumptions of Lemma 3, and moreover every function $f(z)$ holomorphic in $\bar{D}$ can be uniformly approximated in $\bar{D}$ by polynomials, then

$$
V^{*}(z)=u^{*}(z, E, b), \quad z \in \bar{D}
$$

Proof. By our assumptions $\bar{D}$ is polynomially convex. Therefore, $\Phi(z, E, 0)$ $\equiv \Phi(z, \bar{D}, 0)=1$ for $z \in \bar{D}$. Thus

$$
u(z, E, b)=\lim _{\lambda \downarrow 0} \frac{1}{\lambda} \log \Phi(z, E, \lambda b), \quad z \in \bar{D} .
$$

Let $g(z)$ be an arbitrary function holomorphic in $\bar{D}$ such that for some positive integer $v$ we have

$$
|g(z)| \leqq e^{v b(z)}, \quad z \in E .
$$

There is a sequence of polynomials $\left\{P_{k}(z)\right\}$ uniformly convergent to $g(z)$ in $\bar{D}$. We may assume that

$$
\left|P_{k}(z)\right| \leqq|g(z)|, \quad z \in \bar{D}, \quad k=1,2, \ldots
$$

Let the degree of $P_{k}$ be equal to $v_{k}$. We have

Therefore, due to Lemma 6.1

$$
\left|P_{k}(z)\right| \leqq \exp \left[v_{k} \cdot \frac{v}{v_{k}} b(z)\right], \quad z \in E .
$$

whence by (12.1)

$$
\left|P_{k}(z)\right| \leqq \Phi^{v_{k}}\left(z, E, \frac{v}{v_{k}} b\right)=\left[\Phi^{v_{k} / v}\left(z, E, \frac{v}{v_{k}} b\right)\right]^{v},
$$

Then

$$
\frac{1}{v} \log \left|P_{k}(z)\right| \leqq u(z, E, b), \quad z \in \bar{D}, \quad k=1,2, \ldots .
$$

$$
\frac{1}{v} \log |g(z)| \leqq u(z, E, b), \quad z \in \bar{D} .
$$


Since $g(z)$ is an arbitrary holomorphic function satisfying (21), we have by Lemma 3

$$
V^{*}(z) \leqq u^{*}(z)=u^{*}(z, E, b), \quad z \in \bar{D} .
$$

But the function $u^{*}(z)$, being the upper envelope of functions $(1 / v) \log |P(z)|$ where $P(z)$ is a polynomial such that $(1 / v) \log |P(z)| \leqq b(z)$ for $z \in E$, cannot be larger than $V^{*}(z)$ at any point of $D$. The proof is completed.

It will follow from the following theorem that the domain $G$ considered in Lemma 1 cannot be replaced by any larger domain.

THEOREM 2. If the domain D satisfies the assumptions of Lemma 3, then there exist functions $a_{v}(z), v=0,1, \ldots$, holomorphic in $\bar{D}$ such that the series

$$
f(z, w)=\sum_{v=0}^{\infty} a_{v}(z) w^{v}
$$

converges uniformly on $H$ and on any compact subset of $G$, but it diverges at any point outside of $\bar{G}$.

Proof. It follows from the proof of Lemma 3 that there exists a double sequence of functions $g_{k v}(z)$ holomorphic in $\bar{D}$ such that

$$
\left|g_{k v}(z)\right| \leqq \exp \left[v V^{*}(z)\right], \quad z \in \bar{D}, \quad k, v=1,2, \ldots,
$$

and

$$
\exp V^{*}(z)=\left\{\sup _{k}\left\{\limsup _{v \rightarrow \infty}\left|g_{k v}(z)\right|^{1 / v}\right\}\right\}^{*}
$$

Moreover if

$$
V_{k}(z)=\limsup _{v \rightarrow \infty} \frac{1}{v} \log \left|g_{k v}(z)\right|^{1 / v}, \quad z \in \bar{D}
$$

then there exists a countable set $D^{*} \subset D$, everywhere dense in $D$, such that

$$
\lim _{z^{\prime} \rightarrow z_{0},} \sup _{z^{\prime} \in D^{*}} U\left(z^{\prime}\right)=V^{*}\left(z_{0}\right) \text {. }
$$

Arrange the points of $D^{*}$ into a sequence

$$
p_{1}^{\prime}, p_{1}^{\prime}, p_{2}^{\prime}, p_{1}^{\prime}, p_{2}^{\prime}, p_{3}^{\prime}, \ldots
$$

and let $p_{l}, l=1,2, \ldots$, be the $l$ th point of this sequence. For any $l$ there is $n_{l}$ such that

$$
\limsup _{v \rightarrow \infty} \frac{1}{v} \log \left|g_{n \imath v}\left(p_{l}\right)\right|>U\left(p_{l}\right)-\frac{1}{l}, \quad l=1,2, \ldots
$$

Therefore, there is a sequence of positive integers $v_{1}<v_{2}<\ldots$ such that

$$
\frac{1}{v_{l}} \log \left|g_{n i v l}\left(p_{l}\right)\right|>U\left(p_{l}\right)-\frac{2}{l}, \quad l=1,2, \ldots .
$$

It follows from (25), (26) and (28) that

$$
\limsup _{l \rightarrow \infty} \frac{1}{v_{l}} \log \left|g_{n_{l} v_{l}}(z)\right|=U(z), \quad z \in D^{*} .
$$


Therefore, by (27)

$$
\limsup _{l \rightarrow \infty} \frac{1}{v_{l}} \log \left|g_{n_{l} v_{l}}(z)\right|=V^{*}(z), \quad z \in \bar{D} .
$$

By assumption there is a seqence of domains of holomorphy $\left\{D_{v}\right\}$ such that

$$
D_{v} \supset D_{v+1} \supset \bar{D} \text { and } D_{v}+\bar{D} \text {. }
$$

For any $v=1,2, \ldots$ there is the function $\tilde{g}_{v}(z)$ whose domain of existence is $D_{v}$ Let $\max _{(z, w) \in H}\left|\tilde{g}_{v}(z) w^{v}\right|=M_{v}$. The function $g_{v}(z)=1 / M_{v} \tilde{g}_{v}(z)$ is holomorphic in $D_{v}$ and

whence

$$
\left|g_{v}(z) w^{v}\right| \leqq 1, \quad z \in H,
$$

$$
\left|g_{v}(z)\right| \leqq \exp \left[v V^{*}(z)\right], \quad z \in \bar{D}
$$

Denote by $\tilde{a}_{v}(z)$ the $v$ th function of the sequence

$$
{ }_{v_{1}}^{0, \ldots, 0} g_{n_{1} v_{1}}(z), \quad g_{v_{1}+1}(z), \underset{v_{2}-v_{1}-2}{0, \ldots, 0}, g_{n_{2} v_{2}}(z), \quad g_{v_{2}+1}, 0, \ldots .
$$

We claim that the series

$$
\sum_{v=1}^{\infty} a_{v}(z) w^{v}, \quad \text { where } a_{v}(z)=\frac{1}{v^{2}} \tilde{a}_{v}(z), \quad v=1,2, \ldots,
$$

has all the required properties. First of all it follows directly from the construction of $a_{v}(z)$ that the series is uniformly convergent on $H$. Further by (29) and (31) we have

$$
\left\{\limsup _{v \rightarrow \infty} \frac{1}{v} \log \left|a_{v}(z)\right|\right\}^{*}=V^{*}(z) .
$$

Therefore the series (32) is uniformly convergent on any compact subset of $G$ and it is not convergent in a neighborhood of any point $\left(z_{0}, w_{0}\right)$ such that $z_{0} \in D$ and $\left(z_{0}, w_{0}\right) \notin G$. To end the proof it is enough to show that if $\left(z_{0}, w_{0}\right) \in C G$ and $z_{0} \in C \bar{D}$, then the series (32) is divergent. Indeed, by (30) there is some function $a_{v}(z)$ which is not holomorphic at $z_{0}$; therefore, (32) cannot converge at $\left(z_{0}, w_{0}\right)$.

\section{BIBLIOGRAPHY}

1. S. Bergman, Über eine in gewissen Bereichen gültige Integraldarstellung der Funktionen zweier komplexer Variabler, Math. Z. 59 (1934), 76-96, 605-608.

2. - Functions of extended class in the theory of functins of several complex variables, Trans. Amer. Math. Soc. 63 (1948), 523-547.

3. - Kernel functions and extended classes in the theory of functions of complex variables, Colloque sur les fonctions de plusieurs variables, Brussels (1953), 135-197.

4. H. J. Bremermann, On a generalized Dirichlet problem for plurisubharmonic functions and pseudoconvex domains, Trans. Amer. Math. Soc. 91 (1959), 246-276.

5. M. Fekete, Über die Verteilung der Wurzeln bei gewissen algebraischen Gleichungen mit ganzzahligen Koeffizienten, Math. Z. 17 (1923), 228-249. 
6. B. A. Fuchs, Theory of analytic functions of many complex variables, Moscow, 1948. (Russian)

7. J. Gorski, Sur équivalence de deux constructions de la fonction de Green generalisée, Ann. Soc. Polon. Math. 21 (1948), 70-73.

8. M. Inoue, Sur un procédé pour contruire le solution du problème de Dirichlet, Proceedings of the Imperial Academy 14 (1938), 368-372.

9. F. Leja, Sur les séries des polynomes homogenes, Rend. Circ. Mat. Palermo 56 (1932), 419-445.

10. Sur les suites de polynomes, les ensembles fermés et la fonction de Green, Ann. Soc. Polon. Math. 12 (1934), 57-71.

11. - Sur une famille des fonctions harmoniques dans le plan liées a une fonction donnée sur la frontière d'une domaine, Bull. Acad. Polon. Sci. Lettres, Ser. A, Krakow (1936), 79-92.

12. - Sur une classe de fonctions homogènes et les séries de Taylor des fonctions de deux variables, Ann. Soc. Polon. Math. 22 (1949), 45-268.

13. - Une méthode elementaire de resolution du problème de Dirichlet dans le plan, Ann. Soc. Polon. Math. 23 (1950), 230-245.

14. - Theory of analytic functions, Warsaw, 1957. (Polish)

15. - Sur certains suites liées aux ensembles plans et leur application a la représentation conforme, Ann. Polon. Math. 4 (1957), 8-13.

16. __ Problèmes à resondre posés a la conférence, Colloq. Math. 7 (1959), 153.

17. N. A. Sapogov, On best approximation of analytic functions of several complex variables and on series of polynomials, Mat. Sb. (38) (80) 3 (1956), 331-336. (Russian)

18. J. Siciak, Sur la distribution des points extrémaux dans les ensenbles plans, Ann. Polon. Math. 4 (1958), 214-219.

19. - On an extremal function and domains of convergence of series of homogeneous polynomials, Ann. Polon. Math. 10 (1961), 297-307.

20. —_- On function families with boundary, Pacific J. Math. (to appear).

21. - Some applications of the method of extremal points, Thesis, Ann. Polon. Math. (to appear).

22. J. F. Steffenson, Interpolation, Waverly Press, Baltimore, Md., 1927.

23. G. Szegö, Über orthogonal Polynome, die zu einer gegebenen Kurve der komplexen Ebene gehören, Math. Z. 9 (1921), 218-270.

24. H. C. Thacher, Jr., Derivation of interpolation formulas in several independent variables, New York Acad. Sci. 86(3) (1960), 758-775.

25. A. Weil, Sur les séries de polynomes de deux variables complexes, C. R. Acad. Sci. Paris 194 (1932), 1304-1307.

26. - L'integrale de Cauchy et les fonctions des plusieurs variables, Math. Ann. 111 (1935), 178-182.

27. J. L. Walsh, Interpolation and approximation, Waverly Press, Baltimore, Md., 1935.

JAGIELLONIAN UNIVERSITY,

KraKow, POLAND

STANFORD UNIVERSITY,

Stanford, California 